\title{
How to build a cross-impact model from first principles: Theoretical requirements and empirical results
}

\author{
Mehdi Tomas ${ }^{1,2,3}$, Iacopo Mastromatteo ${ }^{3,4}$, and Michael Benzaquen ${ }^{1,3,4}$ \\ ${ }^{1}$ LadHyX UMR CNRS 7646, Ecole Polytechnique, 91128 Palaiseau Cedex, France \\ ${ }^{2}$ CMAP UMR CNRS 7641, Ecole Polytechnique, 91128 Palaiseau Cedex, France \\ ${ }^{3}$ Chair of Econophysics \& Complex Systems, Ecole Polytechnique, 91128 Palaiseau Cedex, France \\ ${ }^{4}$ Capital Fund Management, 23-25, Rue de l'Université 75007 Paris, France
}

April 3, 2020

\begin{abstract}
Cross-impact, namely the fact that on average buy (sell) trades on a financial instrument induce positive (negative) price changes in other correlated assets, can be measured from abundant, although noisy, market data. In this paper we propose a principled approach that allows to perform model selection for cross-impact models, showing that symmetries and consistency requirements are particularly effective in reducing the universe of possible models to a much smaller set of viable candidates, thus mitigating the effect of noise on the properties of the inferred model. We review the empirical performance of a large number of cross-impact models, comparing their strengths and weaknesses on a number of asset classes (futures, stocks, calendar spreads). Besides showing which models perform better, we argue that in presence of comparable statistical performance, which is often the case in a noisy world, it is relevant to favor models that provide ex-ante theoretical guarantees on their behavior in limit cases. From this perspective, we advocate that the empirical validation of universal properties (symmetries, invariances) should be regarded as holding a much deeper epistemological value than any measure of statistical performance on specific model instances.
\end{abstract}

\section{Contents}

1 Introduction

2 Notations

3 Linear models for cross-impact 4

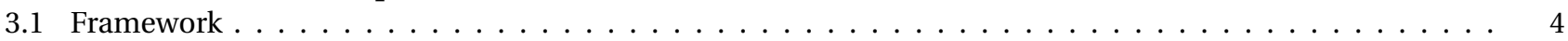

3.2 Covariances and responses $\ldots \ldots \ldots \ldots$

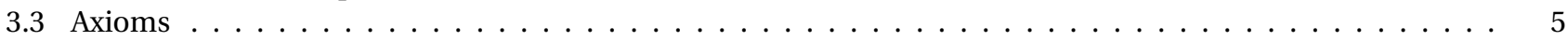

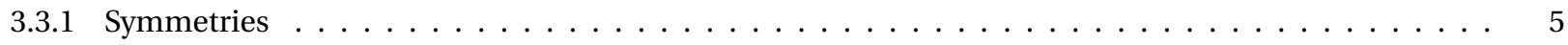

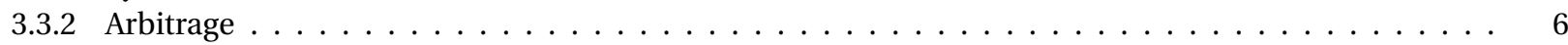

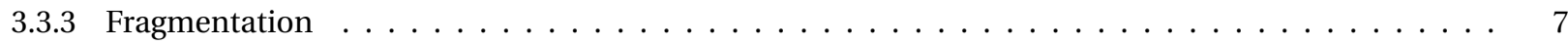

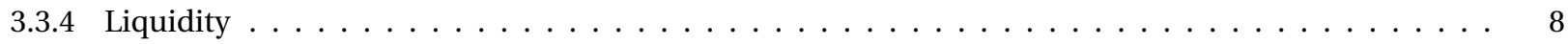

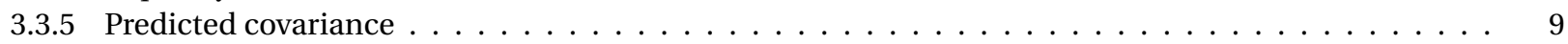

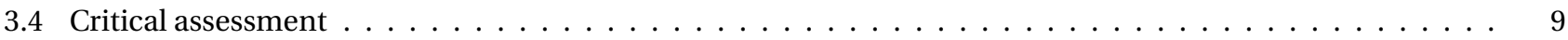

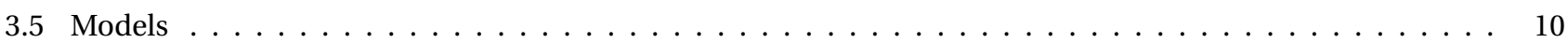

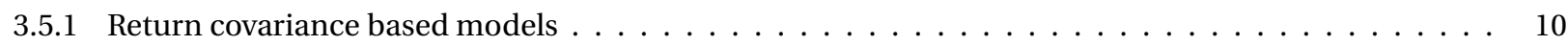

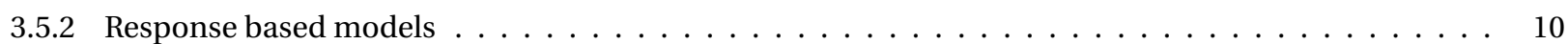

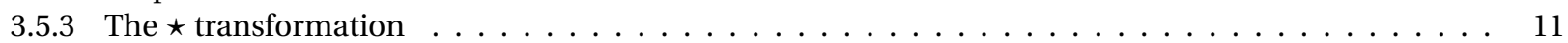

3.6 Relations between axioms and models . . . . . . . . . . . . . . . . . . . . . . 12

4 Empirical results 13

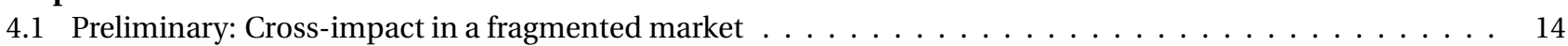

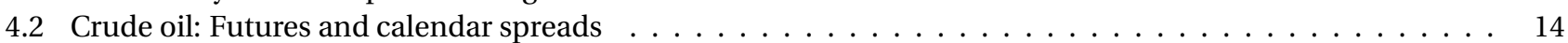

4.3 Bonds and indices $\ldots \ldots \ldots \ldots$

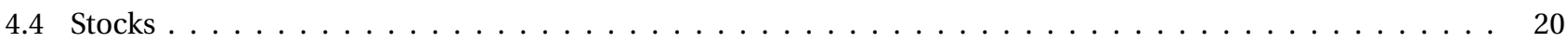




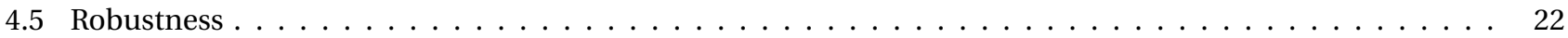

4.5.1 Overfitting and non stationarity in predictive power $\ldots \ldots \ldots$

4.5.2 Influence of the number of instruments and of the timescale of study . . . . . . . . . . . 23

5 Conclusion $\quad 24$

6 Acknowledgments 24

A Models and Axioms $\mathbf{2 6}$

A.1 Relation between fragmentation and liquidity axioms $\ldots \ldots \ldots$

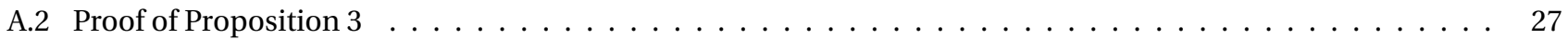

A.3 Proof of Proposition $4 \ldots \ldots \ldots \ldots$

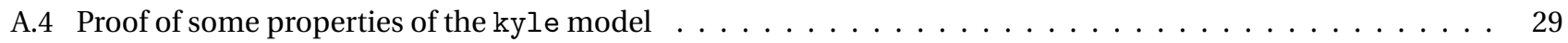

B Proofs of illustrative examples 29

B.1 Proof of Example $1 \ldots \ldots \ldots \ldots$

B.2 Proof of Example $2 \ldots \ldots \ldots \ldots$

\section{Introduction}

The fact that the prices of financial instruments can be moved by trading pressure in the order flow is now a well-established phenomenon, known as Market Impact (see e.g. [2, 4, 19]). In fact, the shape of market impact has been measured in a large number of studies, and displays a structure that is surprisingly robust across assets, time periods and markets. Cross-impact is a multivariate analogue of this market impact, denoting the impact of trading flows from one asset on the price of other, potentially correlated assets. Despite the evidence that cross-impact allows the transmission of information across markets, amplifying shocks in presence of crashes and aggregating liquidity across market venues, the number of empirical results concerning this phenomenon is much more limited (see e.g. [11, 16]). As a relatively new area of research, one can divide empirical studies of cross-impact in two main groups. On the one hand, one finds non-parametric studies focusing on a small number of instruments, and on the other, parametric studies where heuristically-derived models are calibrated on data. Both parametric and non-parametric studies have provided strong evidence for cross-impact. Aside from empirical studies, a number of papers have touched on the subject of cross-impact through other topics, for example via optimal control problems.

We first focus on non-parametric studies. Wang et al. [21, 22] analyzed stocks and used empirical cross-responses between trade signs and prices to build propagator models for cross-impact, using only the direction of the trade (and therefore neglecting the influence of volumes). In [20], the authors define an entropy for impact between stocks: when this coefficient is high, there should be no cross-impact. To study the structure of cross-impact, they build a network which connects assets when the entropy coefficient is low. Thus, a stock in this network with a high ingoing connectivity is affected by trading of many other stocks. On the other hand, trading an asset with a high outgoing connectivity impacts the prices of many other assets. This analysis reveals that the least liquid stocks have high ingoing connectivity and low outgoing connectivity while very liquid stocks have low ingoing connectivity and high outgoing connectivity. In short, the prices of illiquid stocks is driven by the prices of liquid stocks. On the other hand, the paper [17] considered bond markets which, given their higher pairwise correlation, one may expect to show significant cross-impact. Using a similar approach, they fit a propagator model and find strong cross-impact as well as small, but statistically significant, asymmetries.

In [3], the authors fit a different propagator model on stocks. In particular, they find that parametric, factorised models display better performance and are less prone to overfitting than non-parametric alternatives, which gives a strong practical argument for the use of such models. They also found that the first eigenvectors of the stocks return covariance and order flow covariance matrices are roughly aligned: thus the approximation that they commute is partly justified. Naturally this is not always true and it is interesting to know how these models perform when this assumption is violated.

Furthermore, if previous empirical studies yield insight into the shape of one cross-impact model on a given asset class, to our knowledge there is no study allowing to compare different cross-impact models on a variety of markets. Therefore, we are unable to say whether there exists a universal, statistically accurate cross-impact model, or even less ambitiously what are the properties that a cross-impact model should respect in order not to be empirically rejected. 
On the other hand, several theoretical studies have attempted to reduce the universe of possible cross-impact models by constraining their acceptable outcomes. Multivariate propagator-like models were studied in [1] and only a specific class of kernels was shown to satisfy no-arbitrage principles. In particular, the authors showed that a necessary but not sufficient condition for no-arbitrage to hold is that the propagator kernels are positive definite, which implies that non-parametric estimates with asymmetric kernels can be arbitraged (in the absence of linear costs). However, this condition is not sufficiently restrictive to prescribe specific cross-impact models. Interestingly, the non-parametric study [17] calibrates a propagator model on bonds and finds statistically significant asymmetries at different estimation scales (weekly, bi-weekly and monthly) and conservative confidence levels.

If the no-arbitrage property alone is insufficient to determine the structure of cross-impact, one may look at how crossimpact arises in other areas. Two of them are particularly interesting: optimal execution and market making.

In the mean-field framework for optimal execution of [13] (an extension of [6]), a continuum of risk-averse investors seek to acquire a portfolio under a common time horizon. The authors assume that the price of asset $i$ changes linearly with the signed traded volume on asset $i$, which is the sum of the signed traded volume of all investors. While cross-impact is not directly modeled, it appears through the following mechanism. Even though investors are wary of pushing asset prices because it increases their costs, their risk-aversion incentivises them to hedge their target position portfolio by temporarily trading other correlated assets. For example, if an investor wants to acquire 1000 units of asset $i$ which is negatively correlated to asset $j$, his optimal execution schedule will involve, in turn:

(i) buying most of his position on asset $i$ while selling asset $j$

(ii) slowing down his trading speed of asset $i$ and buying back asset $j$ to end the trading session with an inventory of 1000 units of asset $i$ and 0 units of asset $j$.

This point of view interprets cross-impact as the trading pressure generated by opportunistic investors with target positions in correlated financial assets. While this approach provides one explanation of the many possible phenomena underlying cross-impact, it does not provide a recipe one may use on empirical data to estimate the impact induced on asset $i$ by trading asset $j$.

We now turn to the study of cross-impact in the optimal market making literature. In the setting of [10], given an asset dynamics a market maker places quotes away from the mid price, seeking to maximise its expected utility at a terminal date. The probability of the limit orders being executed against an incoming market order depend on the distance to the mid-price. Incoming orders are then modeled as independent Poisson processes. One key assumption is thus that the signed traded order flow of two assets is uncorrelated, contrary to intuition and empirical evidence (see for example in $[3,17,20,21,22])$. Nevertheless, studying a simplified version of the partial differential equation ruling the behaviour of the utility function of the market maker (the link between the solution of the original PDE and simplified PDE is not clear, see [10]), the authors of [8] find that the corrective term to the mark-to-market value of the assets in the utility of the market maker when he holds an inventory $q$ is of the form $-q^{\top} \Lambda q$, where:

$$
\Lambda=D^{-\frac{1}{2}} \sqrt{D^{\frac{1}{2}} \Sigma D^{\frac{1}{2}}} D^{-\frac{1}{2}}
$$

with $\Sigma$ the instantenous return covariance matrix and $D$ playing the role of the typical change of order flow fluctuations around the market-maker's posts. Thus, the risk-averse market maker effectively adjusts the mark-to-market price of the assets to include the estimated liquidation costs of his portfolio. Since we can estimate $D$ and $\Sigma$ on empirical data, we can test this cross-impact model in practice.

While previous works on cross-impact introduced a given model that satisfied some convenient properties (such as [3]), used a specific theoretical framework to derive a model (for example [7]), or focused purely on empirical data (see for example [17, 22], we chose a different approach. In this paper, a cross-impact model is instead simply a function of empirical observables. In place of looking for a single model, we look for reasonable constraints, axioms, which we would like them to satisfy. Data being scarce, the question that we ask ourselves is how should a cross-impact model look like in order to be reasonable?

In particular, we introduce symmetry axioms which we expect any models to satisfy (when abstracting away microstructural effects), fragmentation axioms (generalized from a notion first discussed in [3]), which guarantee suitable behaviour of models when some instruments (or linear combination of instruments) have very small fluctuations, and finally stability axioms to control the impact of trading a basket containing both liquid and illiquid instruments. We further establish 
links between fragmentation and stability axioms and show that, if a model satisfies all symmetry axioms, then it does not display arbitrages in the sense of [1]. These axioms enable us to classify models previously introduced in the literature and give perspective on which may work best in a given scenario.

To test these prescriptions, we apply a variety of cross-impact models to different markets and confirm which axioms are critical to explain empirical observations. We also find that performance of cross-impact models decreases rapidly with the timescale of aggregation of the order flow, increases with the size of the universe of tradeable instruments and generally with the pairwise correlation between instruments.

The paper is organized as follows. Section 2 introduces some notation in force throughout the paper, Section 3 lays down axioms and models, highlighting which axiom each model satisfies. Section 4 presents the calibration results of our zoology of models on different markets. We conclude on the contributions of the paper, remaining open questions and directions for future work in Section 5.

\section{Notations}

Throughout this paper, we write scalars in roman lower cases, vectors in bold lower cases and matrices in roman upper cases. The set of $n$ by $n$ real-valued square matrices is denoted by $\mathscr{M}_{n}$, the set of orthogonal matrices by $\mathscr{O}_{n}$, the set of real symmetric positive semi-definite matrices by $\mathscr{S}_{n}^{+}$and the set of real symmetric positive definite matrices by $\mathscr{S}_{n}^{++}$. Further, given a matrix $A$ in $\mathscr{M}_{n}, A^{\top}$ denotes its transpose. Given $A$ in $\mathscr{S}_{n}^{+}$, we write $A^{1 / 2}$ for a matrix such that $A^{1 / 2}\left(A^{1 / 2}\right)^{\top}=A$ and $\sqrt{A}$ for the matrix square root, the unique positive semi-definite symmetric matrix such that $(\sqrt{A})^{2}=A$. We write $\operatorname{ker}(M)$ for the null space of a matrix $M \in \mathscr{M}_{n}, \Pi_{V}$ for the projector on a linear subspace of $V \in \mathbb{R}^{n}$ and $\bar{\Pi}_{V}=\mathbb{\square}-\Pi_{V}$ for the orthogonal projector. Finally, given a vector $\boldsymbol{v} \in \mathbb{R}^{n}$, we write $\boldsymbol{v}=\left(v_{1}, \ldots, v_{n}\right)$ and $\operatorname{diag}(\boldsymbol{v})$ for the diagonal matrix with diagonal components the components of $\boldsymbol{v}$.

\section{Linear models for cross-impact}

\subsection{Framework}

We are interested in constructing a theory that is able to associate a vector of predicted price changes $\boldsymbol{\Delta} \boldsymbol{p}_{\boldsymbol{t}}=\boldsymbol{p}_{\boldsymbol{t}+\boldsymbol{\Delta t}}-\boldsymbol{p}_{\boldsymbol{t}}=$ $\left(\Delta p_{1, t}, \ldots, \Delta p_{n, t}\right)$ to a signed order flow imbalance $\boldsymbol{q}_{\boldsymbol{t}}=\left(q_{1, t}, \ldots, q_{n, t}\right)$ measured in a time interval $\Delta t$ on a universe of $n$ financial instruments. In order to ensure mathematical tractability of our construction, we focus on the simplest possible scenario, by assuming that (i) the relation between the order flow imbalance $\boldsymbol{q}_{\boldsymbol{t}}$ and the price change $\boldsymbol{\Delta} \boldsymbol{p}_{\boldsymbol{t}}$ is linear, and (ii) the dependence upon past imbalances $\boldsymbol{q}_{t-1}, \boldsymbol{q}_{t-2}, \ldots$ is disregarded. This choice is motivated mainly by the desire of focusing on the most prominent cross-sectional features of these impact models, without overemphasizing the rich structure of the dependence of $\Delta \boldsymbol{p}_{\boldsymbol{t}}$ on the magnitude of the components of $\boldsymbol{q}_{\boldsymbol{t}}$, nor its temporal dynamics. Hence, we will drop the time subscript from both price changes and imbalances from now on. The interested reader is referred to $[3,17,20]$ for more general approaches to this problem. In our stylized setting, the relation between prices and order flows can thus be written as:

$$
\Delta \boldsymbol{p}=\Lambda \boldsymbol{q}+\boldsymbol{\eta},
$$

where $\Lambda$ is the cross-impact matrix, and $\boldsymbol{\eta}=\left(\eta_{1}, \ldots, \eta_{n}\right)$ is a vector of zero-mean random variables independent of $\boldsymbol{q}$. One of the main reasons why practitioners are interested in models of this form is that Eq. (1) allows to build is a predictive theory of impact costs resulting from the execution of a series of trades of size $\boldsymbol{q}$. In particular, if one assumes that the difference between the arrival price and the execution price is given by $\Delta p$, the cost incurred after the execution of a vector of trades $\boldsymbol{q}$ can be written as:

$$
\mathscr{C}(\boldsymbol{q})=\boldsymbol{q}^{\top} \boldsymbol{\Delta} \boldsymbol{p}=\boldsymbol{q}^{\top} \Lambda \boldsymbol{q}+\boldsymbol{q}^{\top} \boldsymbol{\eta} .
$$

Hence, our linear price impact model Eq. (1) induces quadratic impact costs, the average impact cost being given by $\mathbb{E}[\mathscr{C}(\boldsymbol{q})]=\boldsymbol{q}^{\top} \Lambda \boldsymbol{q}$, so that $\Lambda$ quantifies how expensive the trading is on average due to the reaction of the market to the traded flow $\boldsymbol{q}$.

One of the main purposes of this paper is to present a series of prescriptions that can be used to choose the most suitable estimator for the impact matrix $\Lambda$ given a set of empirical observations of market data. The main problem lies in the large number of possible combinations of variables that can be used in order to build $\Lambda$; one would like to have a principled manner to perform model selection. 


\subsection{Covariances and responses}

If one were to consider the traded flows $\boldsymbol{q}$ and the price changes $\boldsymbol{\Delta} \boldsymbol{p}$ as zero-mean Gaussian variables, then it would be very natural to assume that covariances of such observables are sufficient statistics, meaning that no matter what observable one builds, it can always be written as a function of the covariance of such quantities. This leads to the following definition.

Definition 1 (Price and order flow covariances). We define respectively as return covariance, order flow covariance and response the quantities:

$$
\begin{aligned}
\Sigma & :=\mathbb{E}\left[\boldsymbol{\Delta} \boldsymbol{p} \boldsymbol{\Delta} \boldsymbol{p}^{\top}\right] \\
\Omega & :=\mathbb{E}\left[\boldsymbol{q} \boldsymbol{q}^{\top}\right] \\
R & :=\mathbb{E}\left[\boldsymbol{\Delta} \boldsymbol{p} \boldsymbol{q}^{\top}\right] .
\end{aligned}
$$

These quantities appear very naturally in the context of market microstructure, as they capture simple features of the coupled dynamics of prices and order flows. While $\Sigma$ quantifies the co-variation of prices, $\Omega$ captures co-trading of different assets, and $R$ reflects the average change of asset prices with traded order flow.

Though we will not assume price variations nor order flows to be Gaussian random variables, we work under the assumption that the cross-impact matrix $\Lambda$ can be expressed solely as a function of $\Sigma, \Omega$ and $R$. Whereas this is not restrictive in a Gaussian, zero-mean world, in a non-Gaussian context it can be justified as a modeling assumption that allows to capture a large number of distinctive features of the price impact phenomenology while retaining a large degree of simplicity. For convenience, we will note the price volatility $\sigma:=\left(\sqrt{\Sigma_{i i}}\right)_{(1 \leq i \leq n)}$, the signed order flow volatility $\boldsymbol{\omega}:=\left(\sqrt{\Omega_{i i}}\right)_{(1 \leq i \leq n)}$, and the price and flow correlations $\rho:=\operatorname{diag}(\boldsymbol{\sigma})^{-1} \sum \operatorname{diag}(\boldsymbol{\sigma})^{-1}, \rho_{\Omega}:=\operatorname{diag}(\boldsymbol{\omega})^{-1} \Omega \operatorname{diag}(\boldsymbol{\omega})^{-1}$. Though the price volatility $\boldsymbol{\sigma}$ is a familiar quantity, it is worth commenting on the signification of $\omega$. As the average of the signed order flow $\mathbb{E}[\boldsymbol{q}]=0$, $\boldsymbol{\omega}$ quantifies the fluctuations of the traded order flow and will thus be used (and often referred to) as a proxy for liquidity. For example, if asset $i$ is not traded, then $\omega_{i}=0$. These considerations motivate the following definition.

Definition 2 (Cross-impact model). A linear, single period cross-impact model is a function $\Lambda$ of the form

$$
\begin{aligned}
\Lambda: \mathscr{S}_{n}^{+} \times \mathscr{S}_{n}^{++} \times \mathscr{M}_{n} & \rightarrow \mathscr{M}_{n} \\
(\Sigma, \Omega, R) & \mapsto \Lambda(\Sigma, \Omega, R),
\end{aligned}
$$

where we recall that $\mathscr{S}_{n}^{+}$is the space of real, positive semi-definite $n \times n$ matrices, $\mathscr{S}_{n}^{++}$is the space of real, strictly positive definite matrices and $\mathscr{M}_{n}$ the space of $n \times n$ real matrices.

\subsection{Axioms}

Even though writing down the impact matrix $\Lambda$ as a $\Lambda(\Sigma, \Omega, R)$ restricts the possible choices that can be made for modeling it, one still has a large number of degrees of freedom to choose from. This is why we propose an axiomatic approach to the calibration of cross-impact models: instead of comparing models only on the basis of statistical performance, we would like to control ex ante which properties they satisfy. There are three reasons to do this. First, for practical applications it is often preferable to establish theoretical guarantees about the properties satisfied by a cross-impact model. Second, the risk of overfitting in data is considerably reduced when the space of possible models is restricted. And third, it typically has larger scientific value to validate or falsify a generic property of a class of models (symmetry, invariance) with respect to the validation or rejection of a specific instance of a model, for which implementation details might hinder universality.

\subsubsection{Symmetries}

The first properties we review involve the dimensional consistency of the models. First, the ordering used to compute the covariance matrices should be immaterial.

Axiom 1 (Permutational invariance). A cross-impact model is $\Lambda$ is permutational-invariant if, for any permutation matrix $P$, and $(\Sigma, \Omega, R) \in\left(\mathscr{S}_{n}^{+} \times \mathscr{S}_{n}^{++} \times \mathscr{M}_{n}\right)$,

$$
\Lambda\left(P \Sigma P^{\top}, P \Omega P^{\top}, P R P^{\top}\right)=P \Lambda(\Sigma, \Omega, R) P^{\top} .
$$

When the second order statistics are all diagonal, we expect price changes and order flows between distinct assets to be independent in the Gaussian case. Thus, the cross-impact model should respect the independence between assets, which motivates the following axiom. 
Axiom 2 (Direct invariance). A cross-impact model is $\Lambda$ is direct-invariant if, for any $\boldsymbol{\sigma}, \boldsymbol{\omega} \in \mathbb{R}_{+}^{n} \boldsymbol{r} \in \mathbb{R}^{n}$,

$$
\Lambda\left(\operatorname{diag}(\boldsymbol{\sigma})^{2}, \operatorname{diag}(\boldsymbol{\omega})^{2}, \operatorname{diag}(\boldsymbol{r})\right)=\sum_{i=1}^{n} \Lambda\left(\sigma_{i}^{2} \boldsymbol{e}_{\boldsymbol{i}} \boldsymbol{e}_{\boldsymbol{i}}^{\top}, \omega_{i}^{2} \boldsymbol{e}_{\boldsymbol{i}} \boldsymbol{e}_{\boldsymbol{i}}^{\top}, r_{i} \boldsymbol{e}_{\boldsymbol{i}} \boldsymbol{e}_{\boldsymbol{i}}^{\top}\right)
$$

where $\boldsymbol{e}_{\boldsymbol{i}}$ is the $i$-th element of the canonical basis. Furthermore, given that predicted costs are expressed in units of currency, one would expect the currency unit used to express the costs to be immaterial. The next axiom translates this property.

Axiom 3 (Cash-invariance). A cross-impact model $\Lambda$ is cash-invariant if, for any $\alpha>0$, and $(\Sigma, \Omega, R) \in\left(\mathscr{S}_{n}^{+} \times \mathscr{S}_{n}^{++} \times \mathscr{M}_{n}\right)$,

$$
\Lambda\left(\alpha^{2} \Sigma, \Omega, \alpha R\right)=\alpha \Lambda(\Sigma, \Omega, R) .
$$

Similarly, cross-impact models should account for changes in volume units. In equities, one might have stock splits: a company can double the number of outstanding shares and halve their values, though one does not expect the long-term behavior of the system to be affected by this change. This leads to the following axiom.

Axiom 4 (Split invariance). A cross-impact model $\Lambda$ is split-invariant if, for any diagonal matrix of positive elements $D \in \mathscr{M}_{n}$ and $(\Sigma, \Omega, R) \in\left(\mathscr{S}_{n}^{+} \times \mathscr{S}_{n}^{++} \times \mathscr{M}_{n}\right)$

$$
\Lambda\left(D^{-1} \Sigma D^{-1}, D \Omega D, D^{-1} R D\right)=D^{-1} \Lambda(\Sigma, \Omega, R) D^{-1} .
$$

Split invariance guarantees the natural property that impact should adapt to any stock splits, setting aside microstructural effects (tick size effects, lot rounding, etc.).

Another reasonable characteristic is the invariance of the impact model under orthogonal transformations: given that the profit and loss of traders is invariant under this type of transformation (as it is the case in Eq. (2)), it is natural to inquire whether this property is also shared by the corresponding impact model. Again, this abstracts away microstructural effects such as trading fees, bid-ask spread, etc. The following axiom introduces this property.

Axiom 5 (Rotational invariance). A cross-impact model $\Lambda$ is rotation invariant if, for any real orthogonal matrix $O \in \mathscr{O}_{n}$ and $(\Sigma, \Omega, R) \in\left(\mathscr{S}_{n}^{+} \times \mathscr{S}_{n}^{++} \times \mathscr{M}_{n}\right)$

$$
\Lambda\left(O \Sigma O^{\top}, O \Omega O^{\top}, O R O^{\top}\right)=O \Lambda(\Sigma, \Omega, R) O^{\top}
$$

We say of a model which does not satisfy Axiom 5 that it has a privileged basis. Note that any cross-impact model which satisfies Axioms 4 and 5 is invariant under the action of any non-singular matrix $M$.

\subsubsection{Arbitrage}

This family of axioms clarify what properties a cross-impact model should satisfy for costs to be positive on average, or equivalently not to admit any manipulation strategy, in the sense of [9]. The first axiom involves the static arbitrages that it would be possible to exploit in our single-period model if the cost of trading a portfolio of $\boldsymbol{q}$ units, $\mathscr{C}(\boldsymbol{q})=\boldsymbol{q} \boldsymbol{q}^{\top} \boldsymbol{q}$, was negative along some direction.

Axiom 6 (Positive semi-definiteness). The cross-impact model $\Lambda$ takes values in the space of positive semi-definite matrices.

The second axiom that we consider involves dynamic arbitrages in the spirit of [1, 9]. Even though these arbitrages cannot be exploited in our single-period setup, they would emerge by generalizing our setup to the multi-time step setting such as in [17], which is why we choose to also consider this class of arbitrages.

Axiom 7 (Symmetry). The cross-impact model $\Lambda$ takes values in the space of symmetric matrices.

Axioms 6 and 7 together are sufficient to guarantee absence of arbitrages for both linear single-period models $(\mathscr{C}(\boldsymbol{q})=$ $\left.\boldsymbol{q}^{\top} \Lambda \boldsymbol{q}\right)$ and linear multi-period models with factorized kernel $\left(\mathscr{C}(\boldsymbol{q})=\sum_{t, t^{\prime}} \phi\left(t-t^{\prime}\right) \boldsymbol{q}_{t}^{\top} \Lambda \boldsymbol{q}_{t^{\prime}}^{\top}\right)$ : in these cases trading any portfolio will induce a positive cost on average. 


\subsubsection{Fragmentation}

While the previous axioms focused on ruling out strategies with average negative costs, another related issue is the impact of trading assets which have constant prices. Indeed, we expect that if an instrument is a linear combination of other instruments, the cross-impact induced by trading the spread of each instrument should be zero. For example, take a stock traded on multiple markets (say, Apple traded on the Nasdaq and on the Bats venues): for a reasonably large interval of time $\Delta t$, we expect the price on different venues to be the same, or equivalently we expect that it should be impossible to move the combination $p_{\text {Nasdaq }}-p_{\text {Bats. }}$. Thus, buying a volume $q=q_{\text {Nasdaq }}+q_{\text {Bats }}$ of Apple stock should yield the same cost no matter how one fragments the $q_{\text {Nasdaq }}$ units bought on Nasdaq and the $q_{\text {Bats }}$ units bought on Bats. For this reason, this axiom is dubbed fragmentation invariance. We distinguish between three different forms of fragmentation invariance. The first, weak fragmentation invariance, concerns the price changes given by a cross-impact model and is detailed in the next Axiom.

Axiom 8 (Weak fragmentation invariance). The cross-impact model $\Lambda$ is weakly fragmentation invariant if, for any $(\Sigma, \Omega, R) \in$ $\left(\mathscr{S}_{n}^{+} \times \mathscr{S}_{n}^{++} \times \mathscr{M}_{n}\right)$ and $\varnothing \subset V \subseteq \operatorname{ker} \Sigma$,

$$
\Pi_{V} \Lambda(\Sigma, \Omega, R)=0,
$$

where we recall that $\Pi_{V}$ denotes the projector on the linear subspace $V$.

In practice, if a linear combination of prices is assumed not to fluctuate, weak fragmentation invariance guarantees that impact does not produce any volatility in that direction.

Remark 1. From now on, we will implicitly assume that $\operatorname{ker}(\Sigma) \subseteq \operatorname{ker}\left(R^{\top}\right)$, which is consistent with the interpretation of $\Sigma$ and $R$ as covariations in the sense of $E q$. (3). This implies that from the point of view of the fragmentation-related axioms, any condition involving the the kernel of $\Sigma$ will be naturally related to the kernel of $R^{\top}$ as well.

A stronger condition is obtained if one thinks that the volume $\boldsymbol{q}$ traded in directions that do not fluctuate does not have influence on the measured impact. This leads to the following Axiom.

Axiom 9 (Semi-Strong fragmentation invariance). A cross-impact model satisfies semi-strong fragmentation invariance if, besides satisfying the weak fragmentation invariance Axiom 8 , for any $(\Sigma, \Omega, R) \in\left(\mathscr{S}_{n}^{+} \times \mathscr{S}_{n}^{++} \times \mathscr{M}_{n}\right)$ and $\varnothing \subset V \subseteq \operatorname{ker} \Sigma$ one has:

$$
\Lambda(\Sigma, \Omega, R) \Pi_{V}=0 .
$$

One could yet go one step further by stating that the cross-impact model itself should also not depend on how these directions are traded by other market members. This is strong fragmentation invariance, the subject of the next Axiom.

Axiom 10 (Strong fragmentation invariance). An impact model $\Lambda$ is strongly fragmentation invariant if, besides satisfying semi-strong fragmentation invariance (Axiom 9), for any $(\Sigma, \Omega, R) \in\left(\mathscr{S}_{n}^{+} \times \mathscr{S}_{n}^{++} \times \mathscr{M}_{n}\right)$ and $\varnothing \subset V \subseteq \operatorname{ker}(\Sigma)$, one additionally has:

$$
\Lambda(\Sigma, \Omega, R)=\Lambda\left(\Sigma, \bar{\Pi}_{V} \Omega \bar{\Pi}_{V}, R \bar{\Pi}_{V}\right) .
$$

Each of the fragmentation Axioms imposes constrains on the elements of the cross-impact matrix. To summarise, Figure 1 indicates schematically the interplay between all forms of fragmentation invariance.

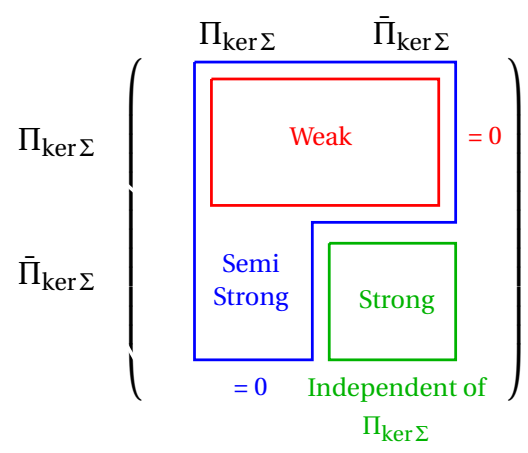

Figure 1: Summary diagram of fragmentation axioms.

We sketched block-wise the cross-impact matrix $\Lambda(\Sigma, \Omega, R)$, separating assets with zero volatility (top left corner) and other assets (bottom right corner). Weak fragmentation invariance (Axiom 8, in red) stipulates that, along the directions of zero price fluctuations and regardless of the traded volumes, the cross-impact model predicts zero price change. Semi-strong fragmentation invariance (Axiom 9, in blue) further requires that there is no impact at all from volume traded along directions of zero price fluctuations. Finally, strong fragmentation invariance (Axiom 10, in green) further stipulates that cross-impact in the directions of non-zero price flucutations is not a function of the volume traded in directions of zero price fluctuations. 


\subsubsection{Liquidity}

A cross-impact model should also have controlled behavior when a set of instruments is considerably less liquid than the rest of the tradable universe. Intuitively, cross-impact models should be such that trading illiquid products does not strongly impact the price of liquid products. We model this by defining a set $V$ of illiquid instruments and by considering the projector $\Pi_{V}$ on the space of such products. Then we can consider the matrix $\bar{\Pi}_{V}+\epsilon \Pi_{V}$ that multiplies by $\epsilon \ll 1$ all the elements belonging to $V$, and consider the modified observables:

$$
\begin{aligned}
\Sigma^{\prime} & =\Sigma \\
\Omega^{\prime} & =\left(\bar{\Pi}_{V}+\epsilon \Pi_{V}\right) \Omega\left(\bar{\Pi}_{V}+\epsilon \Pi_{V}\right) \\
R^{\prime} & =R\left(\bar{\Pi}_{V}+\epsilon \Pi_{V}\right)
\end{aligned}
$$

that correspond to the covariances that one would have measured if the liquidities of instruments belonging to $V$ were to be multiplied by $\epsilon$. We are now ready to formulate axioms relating to how one expects the system to behave when the illiquid instruments are traded.

Axiom 11 (Weak Cross-Stability). We say that a cross-impact model $\Lambda$ is weakly cross-stable if, for any $(\Sigma, \Omega, R) \in\left(\mathscr{S}_{n}^{+} \times\right.$ $\left.\mathscr{S}_{n}^{++} \times \mathscr{M}_{n}\right)$, subspace $V$ and using the above notations, we have:

$$
\bar{\Pi}_{V} \Lambda\left(\Sigma^{\prime}, \Omega^{\prime}, R^{\prime}\right) \Pi_{V} \underset{\epsilon \rightarrow 0}{=} O(1) .
$$

This axiom formulates the intuition that the same notional amount traded on illiquid and liquid products should not move the price of the liquid product by a disproportionate amount. A stronger cross-stability property can also be formulated.

Axiom 12 (Strong Cross-Stability). A cross-impact model $\Lambda$ is strongly cross-stable if, in addition to satisfying weak-cross stability ( Axiom 11), for any $(\Sigma, \Omega, R) \in\left(\mathscr{S}_{n}^{+} \times \mathscr{S}_{n}^{++} \times \mathscr{M}_{n}\right)$, subspace $V$ and using the above notations, one has that:

$$
\bar{\Pi}_{V} \Lambda\left(\Sigma^{\prime}, \Omega^{\prime}, R^{\prime}\right) \bar{\Pi}_{V} \underset{\epsilon \rightarrow 0}{\rightarrow} \bar{\Pi}_{V} \Lambda\left(\bar{\Pi}_{V} \Sigma \bar{\Pi}_{V}, \bar{\Pi}_{V} \Omega \bar{\Pi}_{V}, \bar{\Pi}_{V} R \bar{\Pi}_{V}\right) \bar{\Pi}_{V}
$$

This axiom formalizes the intuition that the price moves on a liquid basket of products induced by trading that very same basket should be independent of the behavior of the illiquid products that have not been traded. Finally, an unresolved question is the effect of trading illiquid instruments on illiquid products. The following axiom deals with this issue.

Axiom 13 (Self-stability). A cross-impact model is self-stable if, using the above notations, we have:

$$
\Pi_{V} \Lambda\left(\Sigma^{\prime}, \Omega^{\prime}, R^{\prime}\right) \Pi_{V} \underset{\epsilon \rightarrow 0}{=} O(1) .
$$

Intuitively this property is less desirable than the previous one: it indicates that, even though a product is illiquid $(q \propto \epsilon$, so that one would expect a diverging impact) the predicted cost of trading such product can be finite, typically because that product is correlated to other more liquid instruments. Figure 2 summarises the differences between weak cross-, strong cross- and self-stability.

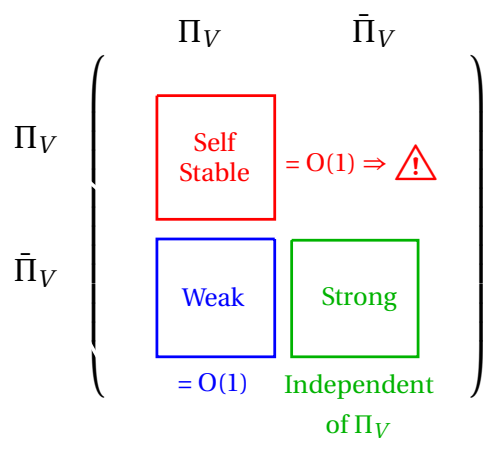

Figure 2: Summary diagram of cross stability properties.

We sketched block-wise the cross-impact matrix $\Lambda(\Sigma, \Omega, R)$, separating illiquid assets (top left corner) and other assets (bottom right corner). The weak cross stable axiom (Axiom 11, in blue) imposes that impact due to trading of illiquid assets is finite. On the other hand, similarly to the strong fragmentation invariance axiom, the strong cross stable axiom (Axiom 12, in green) imposes that cross-impact in the direction of liquid assets does not depend at all on assets with zero liquidity. Finally self-stability (Axiom 13, in red) constrains impact on illiquid assets due to trading of illiquid assets to go to zero. This is a dangerous property as one would fear trading very illiquid instruments would push their price significantly. 


\subsubsection{Predicted covariance}

Finally, it can be interesting to consider whether a cross-impact model predicts a contribution to the return covariance that is proportional to $\Sigma$ or not.

Axiom 14 (Return covariance consistency). A cross-impact model $\Lambda$ is return covariance consistent if, for any $(\Sigma, \Omega, R) \in$ $\left(\mathscr{S}_{n}^{+} \times \mathscr{S}_{n}^{++} \times \mathscr{M}_{n}\right)$, it satisfies (up to a multiplicative constant):

$$
\Sigma=\Lambda(\Sigma, \Omega, R) \Omega \Lambda(\Sigma, \Omega, R)^{\top} .
$$

This axiom is motivated by the fact that under the model in Eq. (1), we expect return covariances to be given by

$$
\Sigma=\mathbb{E}\left[\boldsymbol{\Delta} \boldsymbol{p} \boldsymbol{\Delta} \boldsymbol{p}^{\top}\right]=\Lambda \Omega \Lambda^{\top}+\mathbb{E}\left[\boldsymbol{\eta} \boldsymbol{\eta}^{\top}\right],
$$

so if one assumes that $\mathbb{E}\left[\boldsymbol{\eta} \boldsymbol{\eta}^{\top}\right] \propto \Sigma$ (fundamental return covariance is proportional to the observed one), one would recover return covariance consistency.

\subsection{Critical assessment}

We do not attribute the same level plausibility to the different axioms listed above.

Concerning the invariance-related axioms, we believe the permutational, direct and cash invariance (i.e Axioms 1 to 3 ) to be the most plausible ones, as we don't expect to measure a privileged ordering or price scale in empirical data (tick size effects should be negligible at low enough frequency). Similarly, split invariance (Axiom 4) holds a large degree of plausibility: this symmetry is only expected to be broken by microstructural effects (e.g., lot size). Abstracting away these details, one expects volumes to only appear upon multiplication by prices. Moreover, given that split invariance plays a big role in the deep link between liquidity-related properties and fragmentation-related properties (see section Section 3.6 below), we have a strong a priori in favor of this property. Concerning rotational invariance (Axiom 5), it would not be surprising to find it violated in real markets, given that the physical basis of product is expected to play a privileged role. Though, all invariance-related axioms (Axioms 1 to 5) should be particularly relevant in very liquid markets in which microstructural effects play a limited role and can be abstracted away. Surprisingly, these axioms actually considerably restricts the set of linear cross-impact models.

Weak fragmentation invariance (Axiom 8) is a critical property of a consistent impact model. By construction one does not want to predict price changes along directions that do not fluctuate. For analogous reasons, we believe that even semi-strong and strong fragmentation invariance (Axioms 9 and 10) should be also of crucial importance in order to construct a consistent cross-impact model.

Arbitrage-related axioms Axioms 6 and 7 are of great important in applications, in which one might want to exclude the presence of arbitrages by construction. Still, it is an extremely interesting empirical question to assess whether real markets admit some kind of arbitrage à la [9]. Indeed, even if these axioms were not satisfied in real markets, other mechanisms could prevent statistical arbitrage. For example, transaction costs such as fees or spreads could prevent profitable trading of these arbitrages. Furthermore, it would be surprising to measure a perfectly symmetrical market impact. Instead, one would expect a small asymmetrical component: as long as it is below a certain threshold related to the scale of the symmetrical component, no statistical arbitrages should be possible.

Liquidity-related axioms control the behaviour of cross-impact models to evaluate trading costs. We believe weak-cross stability (Axiom 11) to be a fundamental condition: it should be impossible to move disproportionately the price of liquid assets by trading a moderate amount of illiquid products. The stronger version of this axiom (Axiom 12) is also expected to be fulfilled in data: one expects that liquid products should be insensitive to the behavior of illiquid ones, even in the critical case where they are not traded. On the other hand, self-stability (Axiom 13) can be an undesirable property for applications, because it doesn't penalize the trading of extremely illiquid products.

The return covariance consistency requires that the innovation covariance matrix $\mathbb{E}\left[\boldsymbol{\eta} \boldsymbol{\eta}^{\top}\right]$ is proportional to the return covariance matrix, meaning that the part of the volatility that one can explain with the order flow has the same direction as the total one. There is no ex-ante reason for this to be true, though it is implicitly assumed in the setting of the multivariate Kyle model, see below [7]. Actually, this constraint, combined with no-arbitrage axioms (Axioms 6 and 7) restricts the set of all linear cross-impact models to a single model. 


\subsection{Models}

Now that we have characterized the properties that we can expect from a linear cross-impact model, we provide a set of candidate impact models, whose properties and empirical performance we would like to characterize. We divide these models in two classes; the ones that are based on the return covariance $\Sigma$ and the ones based on the response $R$.

\subsubsection{Return covariance based models}

Let us start with the simplest possible linear impact model: one without cross-impact.

Definition 3 (direct model). The direct model is defined for any $(\Sigma, \Omega, R) \in\left(\mathscr{S}_{n}^{+} \times \mathscr{S}_{n}^{++} \times \mathscr{M}_{n}\right)$ as:

$$
\Lambda_{\text {direct }}(\Sigma, \Omega, R):=\operatorname{diag}(\boldsymbol{\sigma})^{1 / 2} \operatorname{diag}(\omega)^{-1 / 2} .
$$

To generalize this model to the multivariate setting, while respecting cash invariance, weak fragmentation invariance and consistency with correlations, a first idea is to use the matrices $\Sigma^{1 / 2}$ and $\Omega^{-1 / 2}$. Since $\Omega^{-1 / 2} \boldsymbol{q}$ is a whitening transformation, this model is referred to as the whitening model.

Definition 4 (whitening model). Recall that given $M \in \mathscr{S}_{n}^{+}, M^{1 / 2}$ indicates a symmetric matrix factorization $\left(\right.$ i.e., $M^{1 / 2}\left(M^{1 / 2}\right)^{\top}=$ 【). The whitening model $^{1}$ is defined, for any $(\Sigma, \Omega, R) \in\left(\mathscr{S}_{n}^{+} \times \mathscr{S}_{n}^{++} \times \mathscr{M}_{n}\right)$, as:

$$
\Lambda_{\text {whitening }}(\Sigma, \Omega, R):=\Sigma^{1 / 2} \Omega^{-1 / 2} \text {. }
$$

Unfortunately, this estimator does not respect symmetry or positive-definiteness (Axioms 6 and 7), strong fragmentation invariance (Axiom 10) and cross-stability (Axiom 11). To impose symmetry and strong fragmentation invariance, the el model ${ }^{2}$ proposed in [14] defines an impact model directly in the basis of the return covariance matrix, and fixes its eigenvalues in order to be consistent with dimensional analysis. This model then assumes by construction $[\Lambda, \Sigma]=0$, which implies that impact is along the directions of the return covariance matrix.

Definition 5 (el model). The eigenliquidity (el) model is defined, for any $(\Sigma, \Omega, R) \in\left(\mathscr{S}_{n}^{+} \times \mathscr{S}_{n}^{++} \times \mathscr{M}_{n}\right)$, as

$$
\Lambda_{\mathrm{el}}(\Sigma, \Omega, R)=\sum_{a=1}^{n} \boldsymbol{s}_{a} \frac{\sqrt{\lambda_{a}}}{\left(\boldsymbol{s}_{a}^{\top} \Omega \boldsymbol{s}_{a}\right)^{1 / 2}} \boldsymbol{s}_{a}^{\top},
$$

where we have introduced the eigenvalue decomposition of $\Sigma:=\sum_{a=1}^{n} \boldsymbol{s}_{a} \lambda_{a} \boldsymbol{s}_{a}^{\top}$.

The el model is cross-stable, self-stable (Axioms 11 to 13) and is return covariance inconsistent (Axiom 14). As mentioned above, there is in fact only one model which satisfies all the axioms that we have provided: the so-called multivariate kyle model, see [7].

Definition 6 (kyle model). The kyle model is defined, for any $(\Sigma, \Omega, R) \in\left(\mathscr{S}_{n}^{+} \times \mathscr{S}_{n}^{++} \times \mathscr{M}_{n}\right)$, as:

$$
\Lambda_{\mathrm{kyle}}(\Sigma, \Omega, R):=\left(\Omega^{-1 / 2}\right)^{\top} \sqrt{\left(\Omega^{1 / 2}\right)^{\top} \Sigma \Omega^{1 / 2}} \Omega^{-1 / 2} .
$$

The kyle model is extremely similar to the cross-impact mark-to-market adjustment found in [8, 10].

\subsubsection{Response based models}

All the models presented above assume that it is possible to relate the effect of the price to the order flow imbalance with the total return covariance, implicitly assuming that there is no distinction between the directions along which prices respond to liquidity and the ones along which prices fluctuate independently of the order flow. Then, one could expect the response $R=\mathbb{E}\left[\boldsymbol{\Delta} \boldsymbol{p} \boldsymbol{q}^{\top}\right]$ to be more informative in selecting the effect of liquidity shocks, because instead of capturing the totality of the price variations, it is only determined by the ones that are aligned with the order flow $\boldsymbol{q}$. This leads to the following class of models.

First, we can define a response-based direct impact model similar to Eq. (6).

Definition 7 ( $r$-direct model). The response direct ( $r$-direct) model is defined, for any $(\Sigma, \Omega, R) \in\left(\mathscr{S}_{n}^{+} \times \mathscr{S}_{n}^{++} \times \mathscr{M}_{n}\right)$, as:

$$
\Lambda_{\mathrm{r}-\operatorname{direct}}(\Sigma, \Omega, R):=\operatorname{diag}\left(\left(R_{i i}\right)_{i=1}^{n}\right) \operatorname{diag}(\omega)^{-1} .
$$

\footnotetext{
${ }^{1}$ The whitening model is not independent of the symmetric factorization chosen for $\Sigma$ and $\Omega$. As convention, we will take the square root obtained by an orthogonal decomposition of each matrix and the square root of their eigenvalues.

${ }^{2}$ The model proposed in [14] is actually the response-based one, referred later as $\mathrm{r}-\mathrm{el} \star$ model.
} 
This model corresponds to the Maximum-Likelihood Estimator of the cross-impact matrix $\Lambda$ under the constraint $\Lambda_{i j}=0$ for $i \neq j$. Hence, it is quite natural that by trying to replicate the same construction as for the whitening model in the response-based context in order to get a truly multivariate estimator, one obtains the Maximum Likelihood estimator for the matrix $\Lambda$.

Definition 8 (ml model). The maximum likelihood (ml) model is defined, for any $(\Sigma, \Omega, R)$ in $\left(\mathscr{S}_{n}^{+} \times \mathscr{S}_{n}^{++} \times \mathscr{M}_{n}\right)$, as:

$$
\Lambda_{\mathrm{ml}}(\Sigma, \Omega, R):=R \Omega^{-1} \text {. }
$$

The $\mathrm{ml}$ does not satisfy desirable arbitrage or liquidity axioms. Thus, for similar reasons the el was introduced, we introduce a $\mathrm{r}$-el model, so to have a response-based model satisfying more axioms while coinciding with the $\mathrm{ml}$ when $R$ and $\Omega$ commute.

Definition 9 ( $r$-el model). The response-based eigenliquidity $\left(\mathrm{r}\right.$-el) model is defined, for any $(\Sigma, \Omega, R)$ in $\left(\mathscr{S}_{n}^{+} \times \mathscr{S}_{n}^{++} \times \mathscr{M}_{n}\right)$, as:

$$
\Lambda_{\mathrm{r}-\mathrm{el}}(\Sigma, \Omega, R):=\sum_{a} \boldsymbol{s}_{a} \frac{\boldsymbol{s}_{a}^{\top} R \boldsymbol{s}_{\boldsymbol{a}}}{\boldsymbol{s}_{a}^{\top} \Omega \boldsymbol{s}_{a}} \boldsymbol{s}_{a}^{\top},
$$

where $\boldsymbol{s}_{\boldsymbol{a}}$ are the eigenvectors of $\Sigma$. model.

Finally, we can replicate the construction of the kyle estimator in a response-based context, so to obtain the following

Definition 10 (r-kyle model). The response-based Kyle (r-kyle) model is defined, for any $(\Sigma, \Omega, R)$ in $\left(\mathscr{S}_{n}^{+} \times \mathscr{S}_{n}^{++} \times \mathscr{M}_{n}\right)$, as:

$$
\Lambda_{\mathrm{r}-\mathrm{kyle}}(\Sigma, \Omega, R):=\left(\Omega^{-1 / 2}\right)^{\top} \sqrt{\left(\Omega^{1 / 2}\right)^{\top} R \Omega^{-1} R^{\top} \Omega^{1 / 2}} \Omega^{-1 / 2} .
$$

Note that one might intuitively rationalize this model by generalizing the approach of [7] to the case in which a market maker faces insiders whose price information is misaligned with respect to the total price variations, as if not all information about price variation was revealed through trading.

\subsubsection{The $\star$ transformation}

Some of the models defined in the previous section (whitening, el, $r-e l$ ) violate split invariance, due to the fact that the eigenvectors $\left\{\boldsymbol{s}_{a}\right\}_{a=1}^{n}$ of the return covariance $\Sigma$ do not transform like prices under the effect of volume changes, even though they are well-behaved under rotation. Summarizing, these models violate Axiom 4 but respect Axiom 5 . If one is willing to trade one axiom for the other, it is possible to cure the lack of split invariance by introducing a privileged basis, thus sacrificing Axiom 5. One might in fact argue that split invariance should be more fundamental than rotational invariance, given that the basis of physical products should be allowed to play a special role. Trading Axiom 4 with Axiom 5 can be achieved through the following transformation.

Definition 11 (The $\star$ transformation). Given a cross-impact model $\Lambda$, the starred version of $\Lambda$, written $\Lambda^{\star}$, is a cross-impact model defined for any $(\Sigma, \Omega, R)$ in $\left(\mathscr{S}_{n}^{+} \times \mathscr{S}_{n}^{++} \times \mathscr{M}_{n}\right)$ as:

$$
\Lambda^{\star}(\Sigma, \Omega, R):=\operatorname{diag}(\boldsymbol{\sigma}) \Lambda\left(\rho, \Omega^{\star}, R^{\star}\right) \operatorname{diag}(\boldsymbol{\sigma}),
$$

where we have defined $\Omega^{\star}=\operatorname{diag}(\boldsymbol{\sigma}) \Omega \operatorname{diag}(\boldsymbol{\sigma})$ and $R^{\star}=\operatorname{diag}(\boldsymbol{\sigma})^{-1} R \operatorname{diag}(\boldsymbol{\sigma})$, as well as $\rho:=\operatorname{diag}(\boldsymbol{\sigma})^{-1} \Sigma \operatorname{diag}(\boldsymbol{\sigma})^{-1}$ is the return correlation matrix.

In practice, the starred version of a cross-impact model applies the original cross-impact model after rescaling all the observables in units of risk via a multiplication by the volatility $\boldsymbol{\sigma}$. Of course, this transformation has no effect on models that satisfy split invariance.

Table 1 summarises the axioms satisfied by each model. 


\begin{tabular}{|c|c|c|c|c|c|c|c|c|c|c|c|c|c|c|}
\hline \multirow[t]{2}{*}{ Model } & \multicolumn{5}{|c|}{ Symmetries } & \multicolumn{2}{|c|}{ Arbitrage } & \multicolumn{3}{|c|}{ Fragmentation } & \multicolumn{3}{|c|}{ Liquidity } & \multirow{2}{*}{$\begin{array}{c}\text { Covariances } \\
\text { PCC }\end{array}$} \\
\hline & PI & DI & CI & SI & RI & SA & DA & WFI & SSFI & SFI & WCS & SCS & SS & \\
\hline direct & $\checkmark$ & $\checkmark$ & $\checkmark$ & $\checkmark$ & $x$ & $\checkmark$ & $\checkmark$ & $x$ & $x$ & $x$ & $\checkmark$ & $\checkmark$ & $x$ & $x$ \\
\hline whitening & $\checkmark$ & $\checkmark$ & $\checkmark$ & $x$ & $\checkmark$ & $x$ & $x$ & $\checkmark$ & $x$ & $x$ & $x$ & $x$ & $x$ & $\checkmark$ \\
\hline whitening $\star$ & $\checkmark$ & $\checkmark$ & $\checkmark$ & $\checkmark$ & $x$ & $x$ & $x$ & $\checkmark$ & $x$ & $x$ & $x$ & $x$ & $x$ & $\checkmark$ \\
\hline el & $\checkmark$ & $\checkmark$ & $\checkmark$ & $x$ & $\checkmark$ & $\checkmark$ & $\checkmark$ & $\checkmark$ & $\checkmark$ & $\checkmark$ & $\checkmark$ & $\checkmark$ & $\checkmark$ & $x$ \\
\hline el` & $\checkmark$ & $\checkmark$ & $\checkmark$ & $\checkmark$ & $x$ & $\checkmark$ & $\checkmark$ & $\checkmark$ & $\checkmark$ & $\checkmark$ & $\checkmark$ & $\checkmark$ & $\checkmark$ & $x$ \\
\hline kyle & $\checkmark$ & $\checkmark$ & $\checkmark$ & $\checkmark$ & $\checkmark$ & $\checkmark$ & $\checkmark$ & $\checkmark$ & $\sqrt{ }$ & $\checkmark$ & $\checkmark$ & $\checkmark$ & $x$ & $\sqrt{ }$ \\
\hline$r$-direct & $\checkmark$ & $\checkmark$ & $\checkmark$ & $\checkmark$ & $x$ & $\checkmark$ & $x$ & $x$ & $x$ & $x$ & $\checkmark$ & $\checkmark$ & $x$ & $x$ \\
\hline $\mathrm{ml}$ & $\checkmark$ & $\checkmark$ & $\checkmark$ & $\checkmark$ & $\checkmark$ & $x$ & $x$ & $\checkmark$ & $x$ & $x$ & $x$ & $x$ & $x$ & $x$ \\
\hline$r-e l$ & $\checkmark$ & $\checkmark$ & $\checkmark$ & $x$ & $\checkmark$ & $x$ & $\checkmark$ & $\checkmark$ & $\checkmark$ & $\checkmark$ & $\checkmark$ & $\checkmark$ & $\checkmark$ & $x$ \\
\hline$r-e l \star$ & $\checkmark$ & $\checkmark$ & $\checkmark$ & $\checkmark$ & $x$ & $x$ & $\checkmark$ & $\checkmark$ & $\checkmark$ & $\checkmark$ & $\checkmark$ & $\checkmark$ & $\checkmark$ & $x$ \\
\hline r-kyle & $\checkmark$ & $\checkmark$ & $\checkmark$ & $\checkmark$ & $\checkmark$ & $\checkmark$ & $\checkmark$ & $\checkmark$ & $\checkmark$ & $\checkmark$ & $\checkmark$ & $\checkmark$ & $x$ & $x$ \\
\hline
\end{tabular}

Table 1: Summary of axioms satisfied by different cross-impact model. We use the symbol $\boldsymbol{V}$ for axioms that are satisfied and $\boldsymbol{X}$ for axioms that are violated. We use the color green in order to label a desirable property of the model, red for an undesirable property of the model. Yellow is used for properties/models whose violation might not be particularly relevant in order to explain empirical data, although they are interesting to consider. Axioms are grouped by category and the order in which they were presented in the text.

\subsection{Relations between axioms and models}

The properties listed above are not independent, and one can easily derive several relations that can provide further intuition on the axioms above, and additionally relate them to some of the models. It is particularly instructive to relate the fragmentation-related axioms to the liquidity-related ones. Proofs of results presented here are given in Appendix A.

Proposition 1. Let $\Lambda$ be cross-impact model which satisfies split symmetry (Axiom 4) and semi-strong fragmentation invariance (Axiom 9). Then:

(i) $\Lambda$ is weakly cross-stable (Axiom 11) if for a generic linear subspace $V$

$$
\bar{\Pi}_{V} \Lambda\left(\Sigma^{\prime \prime}, \Omega, R^{\prime \prime}\right) \Pi_{V} \underset{\varepsilon \rightarrow 0}{=} O(\epsilon) .
$$

(ii) If, additionally, $\Lambda$ is continuous in the first and third argument strongly fragmentation invariant, then $\Lambda$ is strongly cross-stable (Axiom 12).

(iii) $\Lambda$ is self-stable (Axiom 13) if for a generic linear subspace $V$

$$
\Pi_{V} \Lambda\left(\Sigma^{\prime \prime}, \Omega, R^{\prime \prime}\right) \Pi_{V} \underset{\varepsilon \rightarrow 0}{=} O\left(\epsilon^{2}\right) .
$$

where

$$
\begin{aligned}
& \Sigma^{\prime \prime}=\left(\bar{\Pi}_{V}+\epsilon \Pi_{V}\right) \Sigma\left(\bar{\Pi}_{V}+\epsilon \Pi_{V}\right) \\
& R^{\prime \prime}=\left(\bar{\Pi}_{V}+\epsilon \Pi_{V}\right) R
\end{aligned}
$$

Remark 2. Note that the above proposition introduces some additional technical hypothesis that restrict the speed of convergence of the model around the point in which one asks for fragmentation invariance. In order to satisfy items (i) and (iii) above, when fluctuations are of order $\epsilon$, impact should go to zero at speeds of respectively $\epsilon$ and $\epsilon^{2}$. This is obviously a stronger requirement than a simple fragmentation invariance condition, which is a point-wise property.

Even though a simpler, sufficient condition would guarantee the same property is differentiability, interestingly several models do verify a non-pointwise fragmentation property but lack differentiability (e.g., kyle).

Interestingly, the converse property does not hold, thus indicating that the fragmentation invariance properties play a more fundamental role than the liquidity related axioms. For example, an interesting sufficient condition for semi-strong fragmentation invariance is given below.

Proposition 2. Let $\Lambda$ be a split-invariant, weakly cross-stable and self-stable cross-impact model (Axioms 4, 11 and 13). Then if $\operatorname{ker} \Sigma$ can be generated by the canonical basis, then $\Lambda$ is semi-strongly fragmentation invariant (Axiom 9). 
Within the class of models satisfying strong fragmentation invariance and cross-stability, one can find the el, $r$-el,

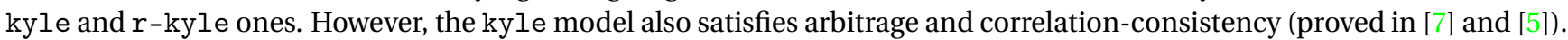
In fact, it is the only model which satisfies these axioms, as discussed in the following proposition, the proof of which is given in Appendix A.2 and heavily inspired by [5, 7].

Proposition 3. Let $\Lambda$ be a symmetric, positive-semidefinite and price-covariance consistent cross-impact model (Axioms 6,7 and 14). Then $\Lambda=\Lambda_{\mathrm{kyle}}$ up to a multiplicative constant.

Hence, there is a single symmetric, positive-semidefinite, correlation-consistent, strongly fragmentation invariant model. Given that the fragmentation-related axioms seem so fundamental, one might wonder how many models one can build that enjoy that family of properties. Indeed, as a trivial corollary of Proposition 1, all models satisfying the above condition are also cross-stable. Surprisingly, the class of models enjoying both split invariance and rotational invariance is quite small: in Appendix A.3 we prove the following proposition.

Proposition 4. A return covariance based cross-impact model $\Lambda$ that is both split invariant and rotational invariant (Axioms 4 and 5) can always be written in the form

$$
\Lambda(\Sigma, \Omega)=\mathscr{L}^{-\top} U F(\boldsymbol{\mu}) U^{\top} \mathscr{L}^{-1},
$$

where

$$
\Omega=\mathscr{L} \mathscr{L}^{\top} \quad ; \quad \hat{\Sigma}:=\mathscr{L}^{\top} \Sigma \mathscr{L} \quad ; \quad U^{\top} \hat{\Sigma} U:=\operatorname{diag}(\boldsymbol{\mu}) \quad ; \quad F(\boldsymbol{\mu}):=\Lambda(\operatorname{diag}(\boldsymbol{\mu}), \text { Ø). }
$$

Furthermore, if $\Lambda$ is cash-invariant and direct-invariant Axioms 2 and 3 , then $F(\boldsymbol{\mu}) \propto \operatorname{diag}(\boldsymbol{\mu})^{1 / 2}$ and $\Lambda=\Lambda \mathrm{kyle}$ up to a multiplicative constant.

The above shows that the only return-based cross-impact model which satisfies all symmetry axioms Axioms 1 to 5 is the kyle model. This indicates that data is bound to play a major role in order to select what cross-impact model is deemed to be more suitable in order to describe market microstructure.

\section{Empirical results}

The focus of the present section is to study a simple set of estimation scenarios for cross-impact models regressed from empirical data. Our goal is to stress test the cross-impact models presented in Section 3. We shall not restrict to ruling out models that appear to be inconsistent with data, but also to establish which of the Axioms presented in Section 3 it is necessary to adopt in order to provide an accurate description of the impact of order flows on prices. To this purpose, we consider three examples constructed with instruments belonging to different asset classes.

(i) First, we consider a set of liquid future contracts traded on the New York Mercantile Exchange (NYMEX) crude oil together with their corresponding calendar-spread contract, in order to illustrate how liquidity aggregates in the presence of very strong correlations. The calendar spread contract is included in the estimation in order to highlight the effect of the presence of a zero-risk mode in the correlation matrix of returns.

(ii) Second, we consider a universe of four contracts, two maturities of a liquid stock index future contract and two maturities of a liquid bond contract. We use this example in order to show how different models react to anticorrelation.

(iii) Finally, we estimate our cross-impact models on a universe of 393 stocks, in order to see how our calibration procedure reacts in the presence of a large number of instruments, so to address the issues of overfitting and robustness to noise.

Our goal is to explain the 1-minute price changes of a basket of instruments using the traded volume during that time span. Price changes are assumed to be independent and identically distributed.

In order to compare the different impact models, we construct three different indicators of performance which emphasize different aspects of prediction errors. All three indicators are parametrized by a symmetric, positive definite matrix $M \in \mathscr{S}_{n}^{+}, M \neq 0$, that is used to construct a generalized $R^{2}$ error for the predicted price changes $\widehat{\boldsymbol{\Delta p}}_{t}$ versus the realized ones $\Delta \boldsymbol{p}_{t}$. In particular, given a realization of the price process $\left\{\Delta \boldsymbol{p}_{t}\right\}_{t=1}^{T}$ of length $T$ and a corresponding series of predictions $\left\{\widehat{\boldsymbol{\Delta p}}_{t}\right\}_{t=1}^{T}$, the generalized $R^{2}$ is defined as:

$$
R^{2}(M):=\frac{\sum_{1 \leq t \leq T}\left(\boldsymbol{\Delta} \boldsymbol{p}_{t}-\widehat{\boldsymbol{\Delta p}}_{t}\right)^{\top} M\left(\boldsymbol{\Delta} \boldsymbol{p}_{t}-\widehat{\boldsymbol{\Delta p}}_{t}\right)}{\sum_{1 \leq t \leq T} \boldsymbol{\Delta} \boldsymbol{p}_{t}^{\top} M \boldsymbol{\Delta} \boldsymbol{p}_{t}} .
$$


Whereas $R^{2}(I)$ is the ordinary $R^{2}$ error, other choices of $M$ can be used in order to highlight different sources of error. In particular we consider:

(i) $M=I_{\sigma}:=\operatorname{diag}(\boldsymbol{\sigma})^{-1}$, to account for errors relative to the typical deviation of the asset considered. This type of error is relevant for strategies predicting idiosyncratic moves of the constituents of the basket, rather than strategies betting on correlated market moves.

(ii) $M=J_{\sigma}:=\left(\Sigma_{i i}^{-1 / 2} \Sigma_{j j}^{-1 / 2}\right)_{1 \leq i, j \leq m}$, to check if the model successfully forecasts the overall direction of all assets, which is obviously relevant for strategies that try to forecast the overall move of the constituents of the basket.

(iii) $M=\Sigma^{-1}$, to consider how well the model predicts the individual modes of the covariance matrix. This would be the relevant error measure for strategies that place a constant amount of risk on the modes of the correlation matrix, leveraging up combinations of products with low volatility and scaling down market direction that exhibit large fluctuations.

Note that the last measure strongly penalizes models violating fragmentation invariance: errors along modes of zero risk should $a$-priori be enhanced by an infinite amount. In this study we have decided to clip the eigenvalues of $\Sigma$ to a small, non-zero amount equal to $10^{-15}$. Given $M \in \mathscr{S}_{n}^{+}, M \neq 0$, we compute scores on empirical data in the following manner. First, we divide data into two subsets of roughly equal length: data from 2016 on the one hand and in 2017 on the other hand. Given data from year $X$ and year $Y$, we calibrate estimators and cross-impact models on year $X$ and use models to predict price changes in year $Y$, writing $R_{X \rightarrow Y}^{2}(M)$ for the average score. In-sample scores are defined as $R_{\mathrm{in}}^{2}(M):=$ $\frac{1}{2}\left(R_{2016 \rightarrow 2016}^{2}(M)+R_{2017 \rightarrow 2017}^{2}(M)\right)$ while out-of-sample scores are defined as $R_{\text {out }}(M):=\frac{1}{2}\left(R_{2016 \rightarrow 2017}^{2}(M)+R_{2017 \rightarrow 2016}^{2}(M)\right)$.

\subsection{Preliminary: Cross-impact in a fragmented market}

Before presenting empirical results, we give a synthetic example to illustrate the behaviour of different cross-impact models. To simplify computations and interpretations of the results, we focus on diagonal volume covariance matrices and general return covariance matrices. Thus, this example deals with correlated assets traded in separate markets. The details of the computations are given in Appendix B.

Example 1 (Two correlated assets on a fragmented market). Let $\Sigma:=\left(\begin{array}{cc}\sigma_{1}^{2} & \sigma_{1} \sigma_{2} \rho \\ \sigma_{1} \sigma_{2} \rho & \sigma_{2}^{2}\end{array}\right)$ and $\Omega:=\left(\begin{array}{cc}\omega_{1}^{2} & 0 \\ 0 & \omega_{2}^{2}\end{array}\right)$ where $\sigma_{1}, \sigma_{2}>0$, $\omega_{1}, \omega_{2} \geq 0$ and $-1 \leq \rho \leq 1$. Then,

$$
\Lambda_{\mathrm{kyle}}(\Sigma, \Omega)=\frac{\sigma_{1} \sigma_{2}}{\sqrt{\sigma_{1}^{2} \omega_{1}^{2}+2 \omega_{1} \omega_{2} \sigma_{1} \sigma_{2} \sqrt{1-\rho^{2}}+\sigma_{2}^{2} \omega_{2}^{2}}}\left(\begin{array}{cc}
\frac{\sigma_{1}}{\sigma_{2}}+\frac{\omega_{2}}{\omega_{1}} \sqrt{1-\rho^{2}} & \rho \\
\rho & \frac{\sigma_{2}}{\sigma_{1}}+\frac{\omega_{1}}{\omega_{2}} \sqrt{1-\rho^{2}}
\end{array}\right) .
$$

Furthermore, if $\sigma_{1}=\sigma_{2}=\sigma$, then:

$$
\begin{aligned}
& \Lambda_{\mathrm{kyle}}(\Sigma, \Omega)=\frac{\sigma}{\sqrt{\omega_{1}^{2}+2 \omega_{1} \omega_{2} \sqrt{1-\rho^{2}}+\omega_{2}^{2}}}\left(\begin{array}{cc}
1+\frac{\omega_{2}}{\omega_{1}} \sqrt{1-\rho^{2}} & \rho \\
\rho & 1+\frac{\omega_{1}}{\omega_{2}} \sqrt{1-\rho^{2}}
\end{array}\right) \\
& \text { and } \Lambda_{\mathrm{e} 1}(\Sigma, \Omega)=\frac{\sigma}{\sqrt{2\left(\omega_{1}^{2}+\omega_{2}^{2}\right)}}\left(\begin{array}{ll}
\sqrt{1+\rho}+\sqrt{1-\rho} & \sqrt{1+\rho}-\sqrt{1-\rho} \\
\sqrt{1+\rho}-\sqrt{1-\rho} & \sqrt{1+\rho}+\sqrt{1-\rho}
\end{array} .\right.
\end{aligned}
$$

Remark 3. When $\sigma_{1}=\sigma_{2}$ in Example 1, we recover an example treated in [7]. Furthermore, as one might expect, if $\sigma_{1}=0$ then trading Asset 1 has no impact on Asset 1's price or on Asset's 2 price: this is naturally related to fragmentation invariance (see Axioms 8 to 10). Similarly, when $\sigma_{1}=\sigma_{2}$ and either $\rho=1$ or $\rho=-1$, the return covariance matrix has only one non-null eigenvalue. As each model satisfies fragmentation invariance, and both coincide in dimension one, they yield the same result.

Remark 4. Example 1 only deals with diagonal volume covariance matrices but invoking basis invariance (Axioms 4 and 5) one can diagonalize $\Omega$ and reduce computations to this example.

\subsection{Crude oil: Futures and calendar spreads}

Description of the dataset We illustrate the performance of the different models by considering a universe of three instruments: two liquid NYMEX Crude Oil future contracts and the corresponding Calendar Spread contract. The first two contracts (respectively, CRUDE0 and CRUDE1) entail an agreement to buy or sell 1000 barrels of oil either at the next 


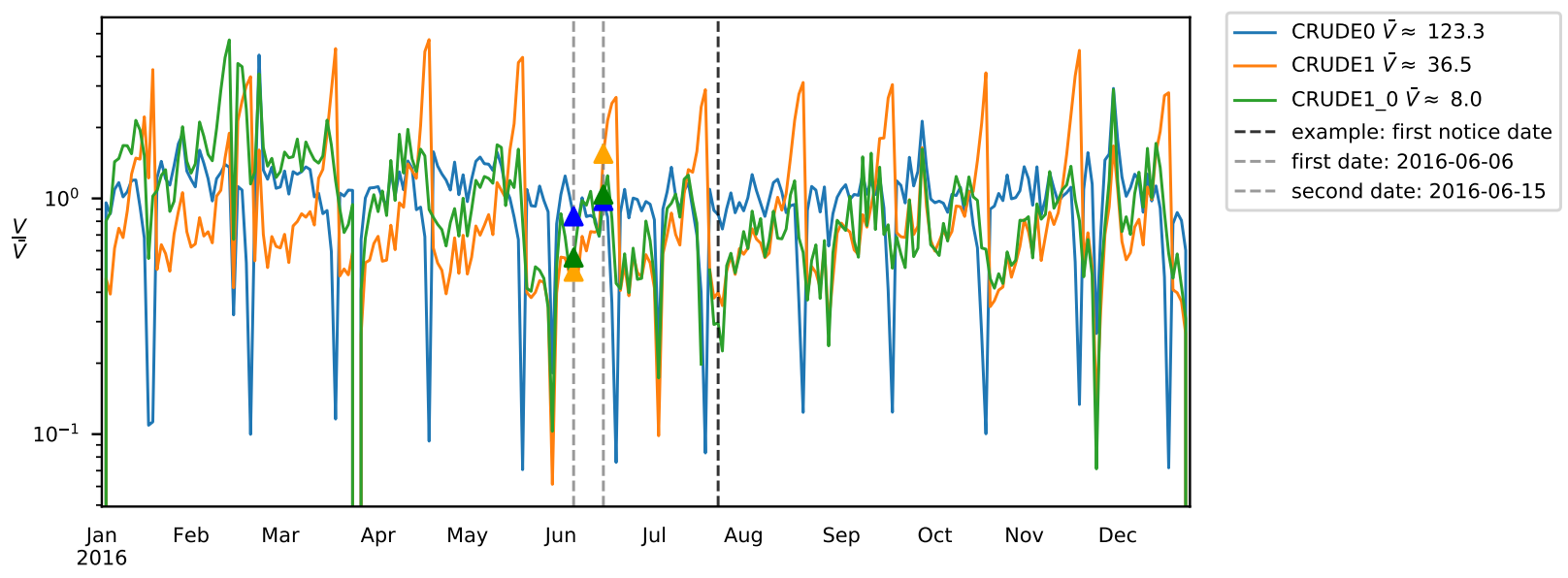

Figure 3: Number of traded NYMEX Crude oil futures and Calendar Spread contracts (in thousands) relative to daily number of traded contracts.

The number of contracts sold relative to the daily average is shown for the front month contract (in blue), the subsequent month (in orange) and the Calendar Spread (in green). The average number of traded NYMEX Crude oil futures and Calendar Spread contracts $\bar{V}$ over 2016 is shown in the upper right corner. Vertical dashed lines show specific dates. An example of first notice date for the front month contract is shown in bold black. After the first notice date, holders of the future contract may ask for physical delivery of the underlying. We also show two dates away from a first notice date: the 6th and 15th of June 2016. Colored triangles show the relative number of contracts exchanged on these dates. Note that the number of contracts is represented in thousands and was not adjusted by the basis point, so that the underlying of each contract is 1000 barrels of oil.

month or at the subsequent month, whereas the Calendar Spread CRUDE1_0 allows to exchange the closer-to-expiry future contract (front month) with a contract settling on the following month. We collected trades and quotes data from January 2016 to December 2017, between 9:30AM to 7:30PM UTC, where most of the trading takes place in our dataset, removing 30 minutes around the opening of trading hours to mitigate intraday seasonality. After filtering and processing, we have a total of 430 days in our sample (237 in 2016 and 193 in 2017). We highlight below two important features of our pre-processing for the estimation of $\Sigma, \Omega$ and $R$.

Pre-processing: accounting for non-stationarity Overall, the front month contract CRUDE0 is by far the most liquid, followed by the subsequent month contract CRUDE1 and the calendar spread CRUDE1_0. However, there are strong seasonal dependencies which are shown in Figure 3. For example, the subsequent month contract becomes more liquid as one approaches the maturity of the front month contract. Global estimators of $\Sigma, \Omega$ and $R$ would thus be biased by this varying liquidity $\boldsymbol{\omega}$ ( $\boldsymbol{\sigma}$ also appears to follow a non-stationary pattern, but is not shown here). Thus, we used local (daily) estimators of price volatility $\boldsymbol{\sigma}_{\boldsymbol{t}}$ and liquidity $\boldsymbol{\omega}_{\boldsymbol{t}}$, and built local covariance estimators $\Sigma_{t}$ and $\Omega_{t}$ by assuming stationarity of the correlations $\varrho=\operatorname{diag}\left(\sigma_{t}\right)^{-1} \Sigma_{t} \operatorname{diag}\left(\sigma_{t}\right)^{-1}$ and $\varrho_{\Omega}=\operatorname{diag}\left(\omega_{t}\right)^{-1} \Omega_{t} \operatorname{diag}\left(\omega_{t}\right)^{-1}$.

Pre-processing: cleaning estimators As illustrated in Figure 4, where the structure of $\Sigma, \Omega$ and $R$ are shown for a typical day, one can appreciate that the correlation between the two future contracts CRUDE0 and CRUDE1 is close to one, whereas the correlation with the Calendar Spread contract is very small, due to the small volatility of the fluctuations along the relative mode. Because of these effects, the sign of the Calendar Spread correlations with CRUDE0 and CRUDE1 is non-trivial to estimate: due to microstructural effects, the measured correlation is dominated by tick-size related effects ${ }^{3}$. In fact, empirical price changes of the Calendar Spread are not given by the difference of price changes of the legs. To solve this issue, we impose the price changes of the Calendar Spread according to the price changes of the futures contracts.

Structure of $\rho, \Omega$ and $R$ The estimators of $\varrho, \Omega$ and $R$ matrices for the 6th of June 2016 are shown in Figure 4. We chose this date as it represents the typical behaviour of these contracts far away from the first notice date, before rolling effects become prevalent. As previously mentioned, the two futures contracts are heavily correlated, which implies that $\Sigma$ will

\footnotetext{
${ }^{3}$ To test this hypothesis, we estimated the empirical smallest eigenvalue of the covariance matrix for multiple futures contract as a function of relative tick size (not shown). If price changes of the Calendar Spread were given by the legs of the contract, this eigenvalue should be equal to zero. However, we found that as the tick size increases, so does the smallest eigenvalue away from zero. This thus validates our hypothesis and justifies the need for additional processing of futures data.
} 

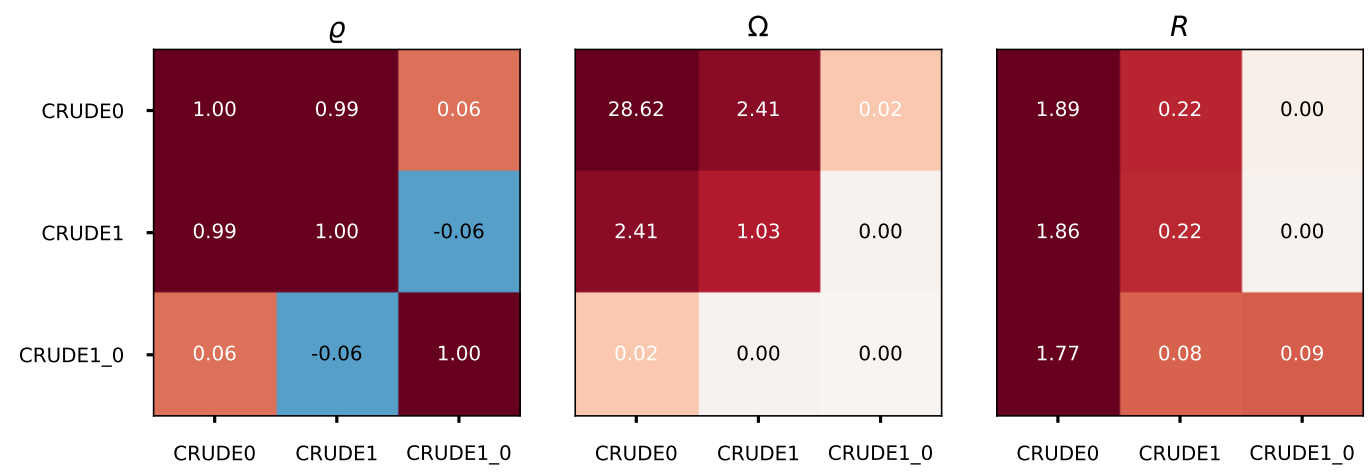

Figure 4: Estimates of $\varrho, \Omega$ and $R$ for Crude contracts (in MUSD).

The return correlation matrix $\rho$ (left), orderflow covariance matrix $\Omega$ (center) and response matrix $R$ (right) were estimated using 2016 data using the procedure described in Section 4.2 and computed on the 6 th of June 2016. To highlight the amount of notional traded, orderflow is reported in millions of exchanged dollars according to the average value of each contract on the 6th of June 2016. Though non-nill, orderflow covariance of Calendar Spread thus appears small because traded notional is much smaller than on each leg of the futures contract.

have one direction of zero fluctuations (due to the Calendar Spread) and another of small, though non-nill fluctuations. Because of these modes of small fluctuations, we expect models which satisfy fragmentation invariance (Axioms 8 to 10) to be preferable. On the other hand, $\Omega$ shows an L-pattern that reflects heterogeneity in liquidity, so that the response shows vertical stripes. This was already noted in [22] in the case of stocks. One should therefore be cautious of models which do not satisfy stability axioms (Axioms 11 to 13), as they will not penalize trading directions of small liquidity.

The empirical structure of $\varrho, \Omega, R$ motivates the following example, where we compare the predictions of models which are both fragmentation invariant but differ in their stability properties.

Example 2 (Calendar Spread and futures). Let $\sigma>0, \omega_{1}, \omega_{2}, \omega_{3} \geq 0,1 \geq \rho \geq-1$ and

$$
\Sigma:=\sigma^{2}\left(\begin{array}{ccc}
1 & \rho & 1-\rho \\
\rho & 1 & -(1-\rho) \\
1-\rho & -(1-\rho) & 2(1-\rho)
\end{array}\right) \quad \Omega:=\left(\begin{array}{ccc}
\omega_{1}^{2} & 0 & 0 \\
0 & \omega_{2}^{2} & 0 \\
0 & 0 & \omega_{3}^{2}
\end{array}\right)
$$

Then, one has:

$$
\Lambda_{\mathrm{el}}(\Sigma, \Omega)=\sqrt{1+\rho} \frac{\sigma}{\bar{\omega}_{1}}\left(\begin{array}{lll}
1 & 1 & 0 \\
1 & 1 & 0 \\
0 & 0 & 0
\end{array}\right)+\sqrt{1-\rho} \frac{\sigma}{\bar{\omega}_{2}}\left(\begin{array}{ccc}
1 & -1 & 2 \\
-1 & 1 & -2 \\
2 & -2 & 4
\end{array}\right),
$$

where $\rho_{\omega}:=\frac{\omega_{1}^{2}-\omega_{2}^{2}}{\sqrt{\left(\omega_{1}^{2}+\omega_{2}^{2}\right)\left(\omega_{1}^{2}+\omega_{2}^{2}+4 \omega_{3}^{2}\right)}}$ and $\bar{\omega}_{1}^{2}:=\frac{\omega_{1}^{2}+\omega_{2}^{2}}{2}, \bar{\omega}_{2}^{2}:=\frac{\omega_{1}^{2}+\omega_{2}^{2}+4 \omega_{3}^{2}}{6}, \lambda_{1}=\sqrt{1+\rho}, \lambda_{2}=\sqrt{1-\rho}$. The lengthy expression of $\Lambda_{\mathrm{kyle}}$ is given in Appendix B.2.

Since it is difficult to analyse the kyle model in full generality of Example 2, we make remarks in special cases of interest.

Remark 5. Once again, when $\rho=1$ there is only one direction of the return covariance matrix with a non-zero eigenvalue (it thus reduces to the rank-one case treated in [7]) and the kyle and el models should coincide (as in the previous example). Indeed, it is easy to check that:

$$
\Lambda_{\mathrm{kyle}}(\Sigma, \Omega) \underset{\rho \rightarrow 1}{=} \frac{\sigma}{\sqrt{\frac{\omega_{1}^{2}+\omega_{2}^{2}}{2}}}\left(\begin{array}{lll}
1 & 1 & 0 \\
1 & 1 & 0 \\
0 & 0 & 0
\end{array}\right) .
$$

This example highlights that $\bar{\omega}_{1}$ plays the role of the effective liquidity of the portfolio $(1,1,0)$. Conversely, when $\rho=-1$, we find:

$$
\Lambda_{\mathrm{kyle}}(\Sigma, \Omega) \underset{\rho \rightarrow-1}{=} \frac{\sigma}{\sqrt{\frac{\omega_{1}^{2}+\omega_{2}^{2}+4 \omega_{3}^{2}}{3}}}\left(\begin{array}{ccc}
1 & -1 & 2 \\
-1 & 1 & -2 \\
2 & -2 & 4
\end{array}\right) .
$$



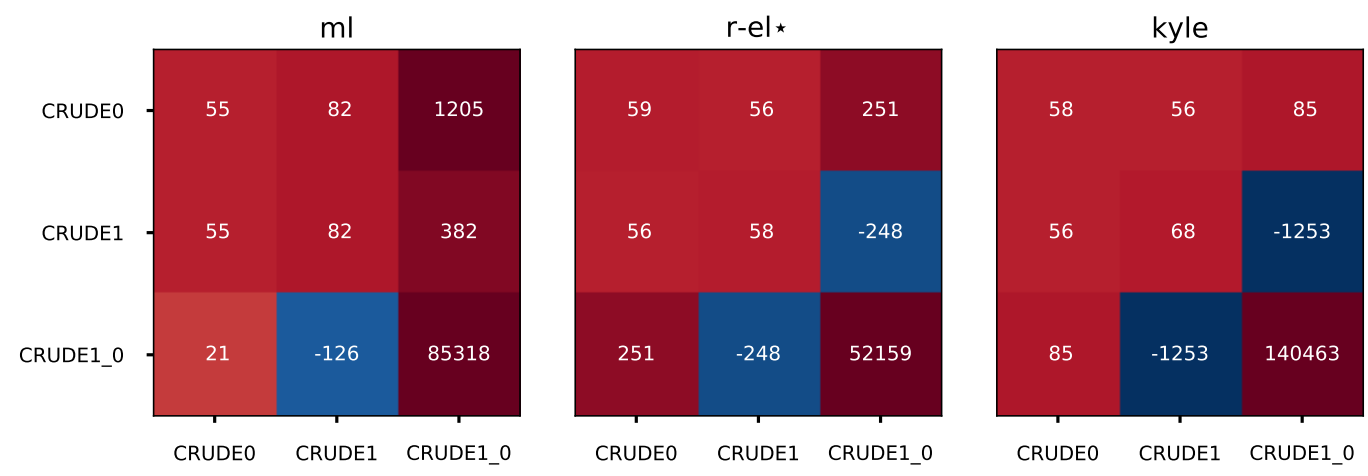

Figure 5: Values of different cross-impact models for Crude contracts.

We report the values of the $\mathrm{ml}$ (left), $r$-el $\star$ (center) and kyle (right) cross-impact models for the covariances of the 6th of June 2016 obtained using the procedure described in Section 4.2. Units are chosen to represent the relative price change in basis points (10 ${ }^{-4}$ of the asset price) by hundred million USD worth of contract traded.

In the $\rho=-1$ regime, buying the calendar spread is equivalent to buying two units of the long leg (or shorting two units of the short leg), which is consistent with the prediction of the kyl e model. As before, we find that $\bar{\omega}_{2}$ plays the role of the effective liquidity of the portfolio $(1,-1,1)$.

Cross impact models for Crude oil contracts For illustrative purposes, we highlight results for a handful of models in Figure 5, selected because of their performance and the different set of axioms which they satisfy: the $\mathrm{ml}, r$-el $\star$ and kyle models. Recall from Table 1 that each of these models satisfies weak fragmentation invariance (Axiom 8) so that one cannot impact directions of zero fluctuations. Therefore, our impact models prevent arbitrage which would trade the physical Calendar Spread contract against the synthetic Calendar Spread (made up of CRUDE0 and CRUDE1). However, models differ in the stability axioms they satisfy. The $\mathrm{ml}$ and kyle models are not self-stable (Axiom 13) while the ml model is. This explains why impact from trading the illiquid Calendar Spread is much larger in the ml and kyle models than in the $r-e l$ model. Overall, by construction, the $r$-el sees a unique basket of liquidity: there is only one direction with a large eigenvalue, the market mode, the relative mode having a much smaller eigenvalue.

Empirical comparison of models Table 2 shows the scores of cross-impact models on the Crude dataset. First, note that models which do not satisfy weak fragmentation invariance (Axiom 8) poorly explain idiosyncratic price changes because of the small volatility of the Calendar Spread. ${ }^{4}$ Furthermore, since $\Sigma$ has one eigenvalue equal to zero, models which do not satisfy weak fragmentation invariance cannot explain risk-weighted price changes. It is therefore more suited to compare all models on the basis of $R^{2}\left(J_{\sigma}\right)$. Variants of direct models account for $33 \%$ and $40 \%$ of the variance of market wide moves. Cross-impact models slightly improve on direct models (scoring around $46 \%$ ). This is somewhat surprising: despite the concentration of liquidity in the front month contract and the large correlation between the front and subsequent month contracts, accounting for the off-diagonal elements of $\Sigma$ and $\Omega$ matters. Finally, among cross-impact models, there is little difference between the performance of the $\mathrm{ml}, \mathrm{r}-\mathrm{el}, \mathrm{r}-\mathrm{el} \star$ and kyle models because the relative mode has very little fluctuations: this three-dimensional system roughly behaves like a one-dimensional system. Overall, this example emphasizes the importance of fragmentation invariance (Axioms 8 to 10) but does not suggest which stability axioms (Axioms 11 to 13) are most relevant. Symmetry axioms may play an important constraining role for return-based covariance models, since the kyle model is the only covariance-based model which yields similar results to the best response-based models. On the other hand, response models which violate some symmetry axioms (such as $r$-el) have good performance. To distinguish between split invariance (Axiom 4) and rotation invariance (Axiom 5), we can compare the scores of models to their starred counterpart. Here, the performance of starred models is close to that of non-starred models. Indeed, taking the example of $r$-el and $r-e l^{*}$, since the liquidity of the calendar spread is thin, the projected liquidity along the three products is the same as the projected liquidity along the two legs of the contract. The same can be said about the projected response. Since the volatility of the two legs are about the same, the first two components of the eigenvectors of $\rho$ and $\Sigma$ should be about the same. We have checked and confirmed this empirically (not shown).

\footnotetext{
${ }^{4}$ For example, though the $R^{2}\left(I_{\sigma}\right)$ score of the $r$-direct model is small, it explains about $35 \%$ of the variance of price changes of CRUDE0, roughly $20 \%$ for CRUDE1 but predicts incorrect price changes for the Calendar Spread CRUDE1_0.
} 


\begin{tabular}{|c|c|c|c|c|c|c|}
\hline \multirow[t]{2}{*}{ Model } & \multicolumn{3}{|c|}{ In-sample } & \multicolumn{3}{|c|}{ Out-sample } \\
\hline & $R_{\text {in }}^{2}\left(I_{\sigma}\right)$ & $R_{\text {in }}^{2}\left(J_{\sigma}\right)$ & $R_{\text {in }}^{2}\left(\Sigma^{-1}\right)$ & $R_{\text {out }}^{2}\left(I_{\sigma}\right)$ & $R_{\text {out }}^{2}\left(J_{\sigma}\right)$ & $R_{\mathrm{out}}^{2}\left(\Sigma^{-1}\right)$ \\
\hline direct & $0.01 \pm 0.01$ & $0.33 \pm 0.01$ & $-\infty$ & $0.01 \pm 0.01$ & $0.33 \pm 0.01$ & $-\infty$ \\
\hline whitening & $0.03 \pm 0.01$ & $0.32 \pm 0.01$ & $-0.05 \pm 0.02$ & $0.04 \pm 0.01$ & $0.32 \pm 0.01$ & $-0.05 \pm 0.02$ \\
\hline whitening & $0.06 \pm 0.01$ & $0.22 \pm 0.01$ & $-0.01 \pm 0.02$ & $0.06 \pm 0.01$ & $0.22 \pm 0.01$ & $-0.01 \pm 0.02$ \\
\hline el & $0.18 \pm 0.01$ & $0.27 \pm 0.01$ & $0.07 \pm 0.02$ & $0.18 \pm 0.01$ & $0.27 \pm 0.01$ & $0.07 \pm 0.02$ \\
\hline el $\star$ & $0.18 \pm 0.01$ & $0.27 \pm 0.01$ & $0.07 \pm 0.02$ & $0.18 \pm 0.01$ & $0.27 \pm 0.01$ & $0.07 \pm 0.02$ \\
\hline kyle & $0.35 \pm 0.01$ & $0.46 \pm 0.01$ & $0.29 \pm 0.02$ & $0.35 \pm 0.01$ & $0.46 \pm 0.01$ & $0.29 \pm 0.02$ \\
\hline$r$-direct & $0.27 \pm 0.01$ & $0.40 \pm 0.01$ & $-\infty$ & $0.27 \pm 0.01$ & $0.40 \pm 0.01$ & $-\infty$ \\
\hline $\mathrm{ml}$ & $0.37 \pm 0.01$ & $0.45 \pm 0.01$ & $0.32 \pm 0.02$ & $0.37 \pm 0.01$ & $0.45 \pm 0.01$ & $0.31 \pm 0.02$ \\
\hline r-el & $0.37 \pm 0.01$ & $0.46 \pm 0.01$ & $0.31 \pm 0.02$ & $0.37 \pm 0.01$ & $0.46 \pm 0.01$ & $0.31 \pm 0.02$ \\
\hline $\mathrm{r}-\mathrm{el} \star$ & $0.37 \pm 0.01$ & $0.46 \pm 0.01$ & $0.31 \pm 0.02$ & $0.37 \pm 0.01$ & $0.46 \pm 0.01$ & $0.31 \pm 0.02$ \\
\hline r-kyle & $0.22 \pm 0.01$ & $0.31 \pm 0.01$ & $0.16 \pm 0.02$ & $0.22 \pm 0.01$ & $0.31 \pm 0.01$ & $0.16 \pm 0.02$ \\
\hline
\end{tabular}

Table 2: In-sample and out-sample scores for Crude contracts.

We reported as $\infty$ the scores of models which are numerically infinite, but due to clipping appear finite.

\subsection{Bonds and indices}

The previous dataset on Crude futures and Calendar Spreads showed the importance of satisfying fragmentation invariance (Axiom 8). However this market corresponds to a pathological case where $\Sigma$ has an eigenvalue equal to zero. Furthermore, we were unable to study the varying liquidity of each contract since the high correlation between calendar spreads left $\Sigma$ with only one large non-nill eigenvalue, so that cross-impact models gave similar results. We now focus on studying the heterogeneity in liquidity in a basket of instruments, using bonds and indices data.

Description of the dataset We look at 10-year US Treasury note futures and the E-MINI futures. We collect data from the Chicago Mercantile Exchange and use the first two upcoming maturities of both contracts (respectively called SPMINI and SPMINI3 for E-MINI contracts and 10USNOTE and 10USNOTE3 for 10-year US treasury notes). E-Mini futures are quarterly, financially settled contracts with maturities in March, June, September and December. At expiry, the final settlement price of E-MINI futures is a proxy for the S\&P500 index using the opening prices of the underlying stocks belonging to the index. Similarly, the 10-year treasury note futures are quaterly, financially settled contracts with maturities in March, June, September and December. At expiry, the final settlement price is volume weighted average price of past trades on the underlying treasury note. ${ }^{5}$ We collected trades and quotes data from January 2016 to December 2017, between 9AM to 7PM UTC, where most of the trading takes place in our dataset. After filtering days for which data for one product was missing, we keep a total of 160 days (75 in 2016 and 85 in 2017). We highlight below one important pre-processing step for the estimation of $\Sigma, \Omega$ and $R$.

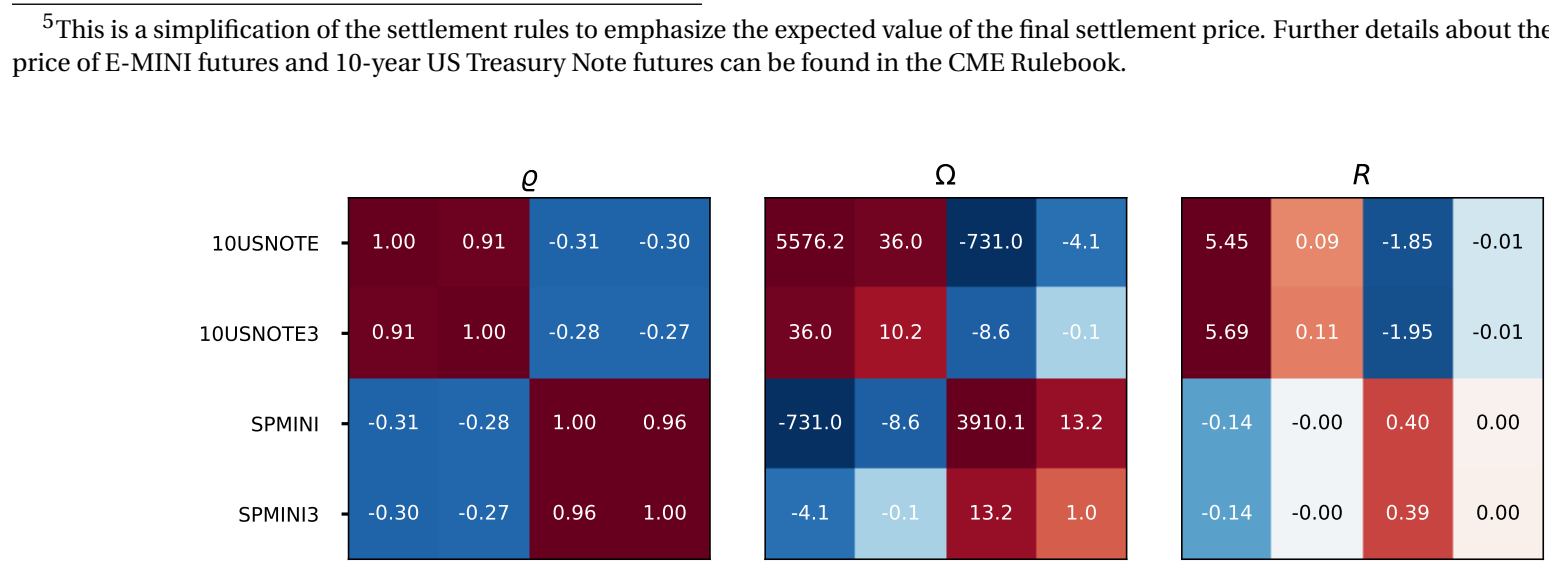

Figure 6: Estimates of $\varrho, \Omega$ and $R$ for bonds and indices (in MUSD).

The return correlation matrix $\rho$ (left), orderflow covariance matrix $\Omega$ (center) and response matrix $R$ (right) were estimated using 2016 data using the procedure described in Section 4.3 and computed on the 17th of August 2016. To highlight the amount of notional traded, orderflow is reported in millions of exchanged dollars according to the average value of each contract on the 17th of August 2016. Basis points were accounted for, so that one traded unit of the futures contracts entitles the owner to one unit of the underlying. 

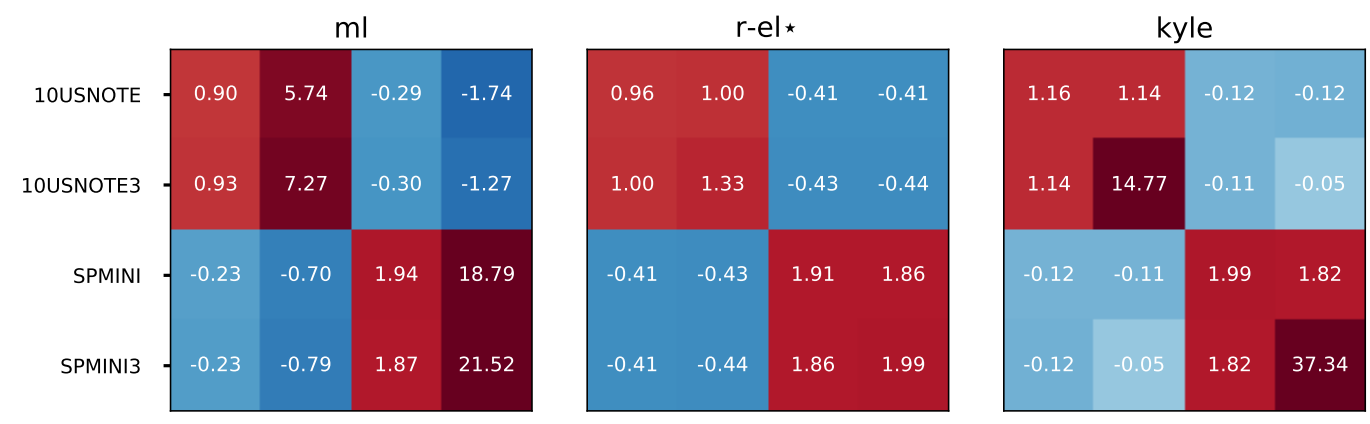

Figure 7: Values of different cross-impact models for bonds and indices.

We report the values of the $\mathrm{ml}$ (left), $r$-el $\star$ (center) and kyle (right) cross-impact models for the covariances of the 17th of August 2016 obtained using the procedure described in Section 4.3. Units are chosen to represent the relative price change in basis points $\left(10^{-4}\right.$ of the asset price) by hundred million USD worth of contract traded.

Pre-processing: accounting for non-stationarity The same non-stationary behaviour observed for Crude Oil futures contract is observed here. Thus we adopt the same estimation procedure for the local covariance estimators $\Sigma_{t}$ and $\Omega_{t}$ by assuming stationarity of the correlations $\varrho=\operatorname{diag}\left(\boldsymbol{\sigma}_{t}\right)^{-1} \sum_{t} \operatorname{diag}\left(\boldsymbol{\sigma}_{t}\right)^{-1}$ and $\varrho_{\Omega}=\operatorname{diag}\left(\boldsymbol{\omega}_{t}\right)^{-1} \Omega_{t} \operatorname{diag}\left(\boldsymbol{\omega}_{t}\right)^{-1}$.

Structure of $\rho, \Omega$ and $R$ The estimators of $\varrho, \Omega$ and $R$ matrices for the 17th of August 2016 are shown in Figure 6. As we expected, contracts with similar underlying are strongly correlated, thus $\rho$ shows 2 by 2 blocks of strongly correlated contracts and an anti-correlation between bonds and futures. The correlation between two contracts is smaller than in the previous example of Crude contracts. This may be because the maturities for Crude contracts were one month apart while the maturities for the bonds or indices futures studied here are 3 months apart. Similarly to the previous example, liquidity is heterogeneous. Non-front month contracts have small liquidity. Interestingly, the structure of the volume covariance matrix also shows anti-correlation between bonds and indices. In this configuration, the discriminating factor between models should be stability axioms (Axioms 11 to 13) rather than fragmentation axioms (Axioms 8 to 10).

Cross impact models for bonds and indices For illustrative purposes, we highlight results for a handful of models in Figure 7, selected because of their performance and the different set of axioms which they satisfy: the $\mathrm{ml}, \mathrm{r}-\mathrm{el} \star \mathrm{and} \mathrm{kyle}$ models. Recall that the $\mathrm{r}-\mathrm{el}$ and kyle models are weakly cross-stable (Axiom 11) while the ml model is not. Thus the ml assigns large impact to less liquid contracts, 10USNOTE3 and SPMINI3. Similarly, the self-stability (Axiom 13) of $r$-el explains the small impact predicted if one trades illiquid contracts. Reassuringly, all models correctly capture the negative index-bonds correlation.

Empirical comparison of models Table 3 shows the scores of cross-impact models on the bonds and indices dataset. The notable difference in the structure of $\Sigma$ between the previous basket of instruments and this set of instruments is the importance of both the market (indices minus bonds) and relative (indices + bonds) modes. In the case of Crude

\begin{tabular}{|c|c|c|c|c|c|c|}
\hline \multirow[t]{2}{*}{ Model } & \multicolumn{3}{|c|}{ In-sample } & \multicolumn{3}{|c|}{ Out-sample } \\
\hline & $R_{\text {in }}^{2}\left(I_{\sigma}\right)$ & $R_{\mathrm{in}}^{2}\left(J_{\sigma}\right)$ & $R_{\text {in }}^{2}\left(\Sigma^{-1}\right)$ & $R_{\text {out }}^{2}\left(I_{\sigma}\right)$ & $R_{\text {out }}^{2}\left(J_{\sigma}\right)$ & $R_{\text {out }}^{2}\left(\Sigma^{-1}\right)$ \\
\hline direct & $-0.11 \pm 0.02$ & $0.09 \pm 0.02$ & $-7.24 \pm 0.21$ & $-0.11 \pm 0.02$ & $0.09 \pm 0.02$ & $-7.23 \pm 0.21$ \\
\hline whitening & $0.03 \pm 0.02$ & $-0.09 \pm 0.03$ & $-0.37 \pm 0.04$ & $0.03 \pm 0.02$ & $-0.10 \pm 0.03$ & $-0.37 \pm 0.04$ \\
\hline whitening $\star$ & $0.05 \pm 0.02$ & $-0.05 \pm 0.03$ & $-0.36 \pm 0.04$ & $0.04 \pm 0.02$ & $-0.05 \pm 0.03$ & $-0.36 \pm 0.04$ \\
\hline el & $0.19 \pm 0.01$ & $0.09 \pm 0.02$ & $-0.26 \pm 0.03$ & $0.19 \pm 0.01$ & $0.09 \pm 0.02$ & $-0.26 \pm 0.03$ \\
\hline el $\star$ & $0.02 \pm 0.02$ & $-0.21 \pm 0.03$ & $-0.37 \pm 0.03$ & $0.02 \pm 0.02$ & $-0.21 \pm 0.03$ & $-0.37 \pm 0.03$ \\
\hline kyle & $0.38 \pm 0.01$ & $0.29 \pm 0.02$ & $0.11 \pm 0.03$ & $0.38 \pm 0.01$ & $0.29 \pm 0.02$ & $0.11 \pm 0.03$ \\
\hline$r$-direct & $0.23 \pm 0.01$ & $0.27 \pm 0.02$ & $-1.69 \pm 0.05$ & $0.23 \pm 0.01$ & $0.27 \pm 0.02$ & $-1.71 \pm 0.05$ \\
\hline $\mathrm{ml}$ & $0.40 \pm 0.01$ & $0.30 \pm 0.02$ & $0.20 \pm 0.03$ & $0.40 \pm 0.01$ & $0.30 \pm 0.02$ & $0.20 \pm 0.03$ \\
\hline r-el & $0.38 \pm 0.01$ & $0.29 \pm 0.02$ & $0.19 \pm 0.03$ & $0.38 \pm 0.01$ & $0.29 \pm 0.02$ & $0.19 \pm 0.03$ \\
\hline$r-e l \star$ & $0.27 \pm 0.01$ & $0.17 \pm 0.02$ & $0.13 \pm 0.03$ & $0.27 \pm 0.01$ & $0.17 \pm 0.02$ & $0.13 \pm 0.03$ \\
\hline r-kyle & $0.25 \pm 0.01$ & $0.14 \pm 0.02$ & $0.07 \pm 0.03$ & $0.24 \pm 0.01$ & $0.14 \pm 0.02$ & $0.07 \pm 0.03$ \\
\hline
\end{tabular}

Table 3: In-sample and out-sample scores for bonds and indices. 
contracts, the relative mode (the price difference of CRUDE0 and CRUDE1) had little to no fluctuations. Here however, one can easily check that the impact contribution of the market and relative modes are comparable. This explains why cross-impact models achieve much better performance relative to direct models to explain idiosyncractic price moves (as can be seen by comparing $R^{2}\left(I_{\sigma}\right)$ scores). On the other hand, the notable difference in the structure of $\Omega$ is the presence of two instruments with very small liquidity. However, there is no clear difference in scores of models according to which stability axioms (Axioms 11 to 13) they satisfy. A second order characteristic of $\Sigma$ is the presence of directions of small fluctuations: the price difference between 10USNOTE and 10USNOTE3 or between SPMINI and SPMINI3. As before, these directions of small fluctuations penalise models which do not respect fragmentation invariance, as evidence by $R^{2}\left(\Sigma^{-1}\right)$ scores. To distinguish between split invariance (Axiom 4) and rotation invariance (Axiom 5), we can compare the scores of models to their star counterpart. Contrary to the previous example, we find here that starred models perform worse than their non-starred counterparts. This suggests, surprisingly, that Axiom 5 may be more relevant than Axiom 4 to explain price changes.

\subsection{Stocks}

The previous datasets emphasized again the importance of satisfying fragmentation invariance (Axioms 8 to 10) but did not yield a clear conclusion on the role of stability axioms (Axioms 11 to 13). However, in both examples the return covariance matrix had directions of small fluctuations because of high pairwise correlation among some instruments. To circumvent this issue, we study the behaviour of cross-impact models in the low-correlation, many assets regime, using stocks data.

Description of the dataset We selected 393 different stocks among the constituents of the S\&P500 and collected trades and quotes data between 2PM and 9:30PM UTC, removing the beginning and end of the trading period to focus on the intraday behaviour of liquidity and volatility and circumvent intraday non-stationary issues. We collected trades and quotes data from January 2016 to December 2017, between 2PM and 9:30PM UTC, to focus on the intraday behaviour of liquidity and volatility and circumvent intraday non-stationary issues. After filtering days for which data for one product was missing, we keep a total of 302 days (154 in 2016 and 148 in 2017). Some summary characteristics of our sample are presented in Table 4.

\begin{tabular}{lccc} 
& \multicolumn{3}{c}{ Quantile } \\
\cline { 2 - 4 } & $10 \%$ & $50 \%$ & $90 \%$ \\
Relative tick size (in \%) & 1.6 & 2.5 & 4.6 \\
Number of trades per day (in thousands) & 5.9 & 12.6 & 29.4 \\
Daily turnover (in MUSD) & 28.5 & 56.1 & 116.2
\end{tabular}

Table 4: Summary statistics for our sample of stocks.
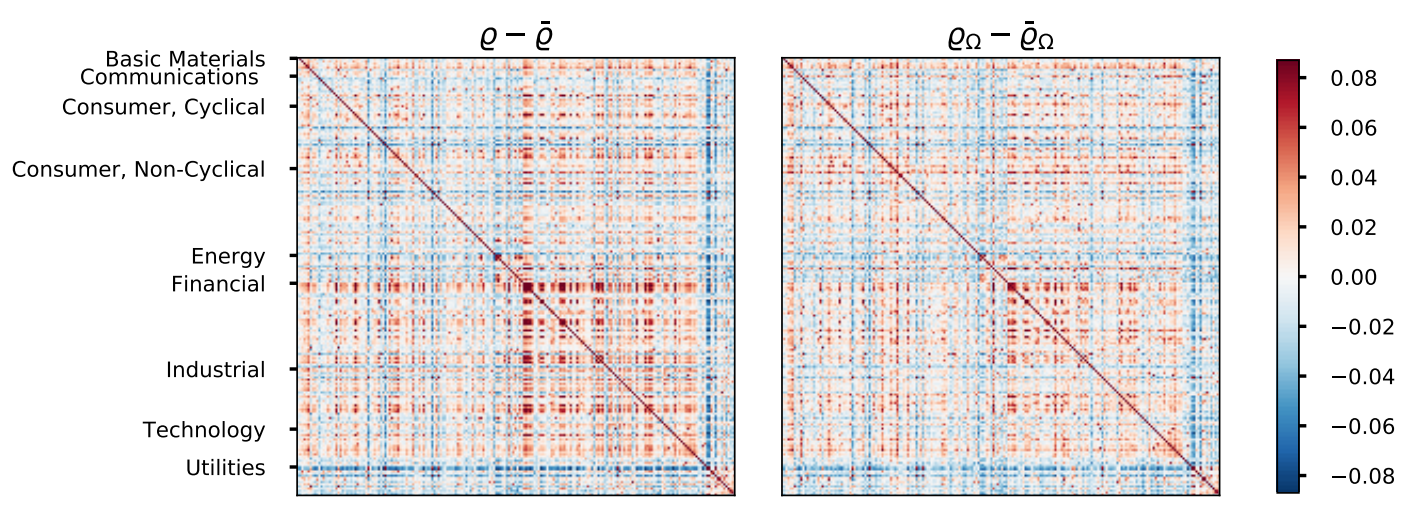

Figure 8: Estimated price and orderflow correlation matrices $\varrho, \varrho_{\Omega}$ for stocks.

We represent the return correlation matrix $\rho$ (left), orderflow correlation matrix $\varrho_{\Omega}$ (right) estimated on 2016. To highlight the amount of notional traded, orderflow is reported in millions of exchanged dollars according to the average value of each contract on the 17 th of August 2016. Correlation matrices were represented instead of covariance matrices due to the large volume heterogeneities between stocks. Stocks were grouped by sectors to highlight the blockwise structure of these matrices. 

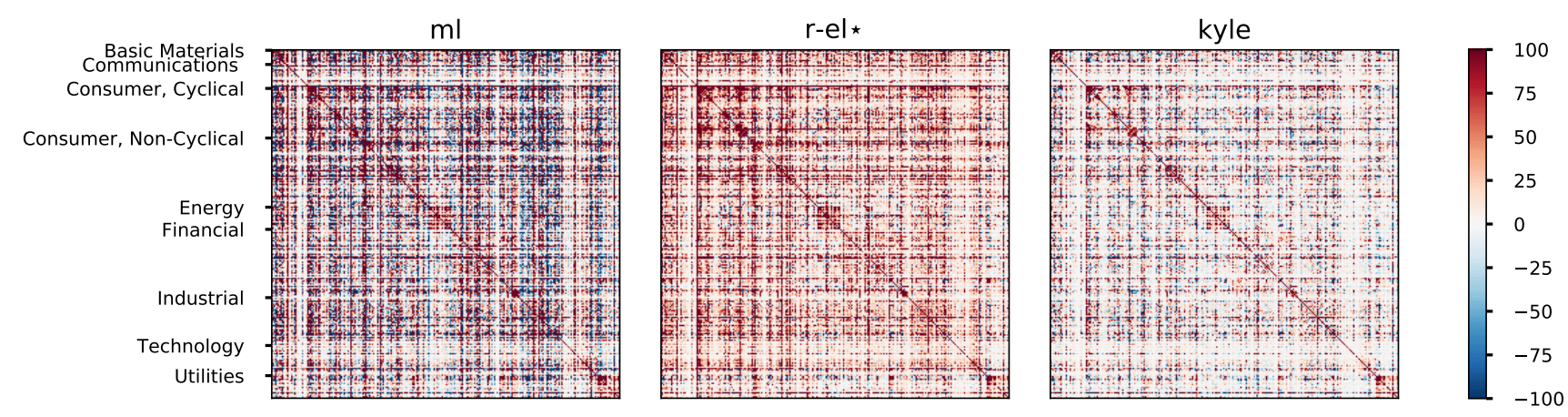

Figure 9: Values of different cross-impact models for stocks.

We report the values of the $\mathrm{ml}$ (left), $r$-el $\star$ (center) and kyle (right) cross-impact models for covariances obtained using the procedure described in Section 4.4. Units are chosen to represent the relative price change in basis points $\left(10^{-4}\right.$ of the asset price) by hundred million USD worth of instruments traded.

Structure of $\varrho, \varrho_{\Omega}$ and $R$ Estimators of $\varrho, \varrho_{\Omega}$ are shown in Figure 8 . To highlight the blockwise structure of these matrices, we show correlations instead of covariances. For the same reasons, $R$ is not shown but presents a bandwise structure one expects from heterogeneities in liquidity. As previously mentioned, pairwise price and orderflow correlations between assets are small, so that the improvement of cross-impact models over direct models should be lower than in previous applications. For more details about the structure of the price and volume covariance matrices, see [3].

Cross impact models for stocks For illustrative purposes, we highlight results for a handful of models in Figure 9, selected because of their performance and the different set of axioms which they satisfy: the $\mathrm{ml}, \mathrm{r}-\mathrm{el} \star$ and kyle models. At first glance, each model appears to present a blockwise structure similar to that of $\varrho, \varrho_{\Omega}$. However, the ml model does not satisfy weak cross-stability (Axiom 11) and thus predicts large impact on liquid stocks if one trades illiquid stocks. By construction the $r-e l \star$ model weighs most impact on the market mode. Finally, the kyle model looks like a symmetrized version of the $r$-el model.

Empirical comparison of models Table 5 shows the scores of cross-impact models on the stocks dataset. Contrary to the two previous examples where only a small number of directions had notable impact contributions, the low pair-wise correlation of stocks (aside from market mode contributions) suggests that there are many directions which contribute to the overall impact. Precisely because of the importance of the market mode, the scores reported in Table 5 strikingly show that cross-impact models can explain market-wide moves up to twice as well as direct models, as shown by the difference in $R^{2}\left(J_{\sigma}\right)$ scores. Naturally, explaining idiosyncratic or eigenportfolio price changes is a more challenging task, but cross-impact models $(\mathrm{r}-\mathrm{el}, \mathrm{r}-\mathrm{el} \star, \mathrm{kyle}, \mathrm{ml})$ improve $\mathrm{r}$-direct scores by $20 \%$ to $30 \%$. To distinguish between split invariance (Axiom 4) and rotation invariance (Axiom 5), we can compare the scores of models to their star counterpart. We find, in continuity with our results on the bonds and indices dataset, that starred models perform better than their

\begin{tabular}{|c|c|c|c|c|c|c|}
\hline \multirow[t]{2}{*}{ Model } & \multicolumn{3}{|c|}{ In-sample } & \multicolumn{3}{|c|}{ Out-sample } \\
\hline & $R_{\text {in }}^{2}\left(I_{\sigma}\right)$ & $R_{\text {in }}^{2}\left(J_{\sigma}\right)$ & $R_{\text {in }}^{2}\left(\Sigma^{-1}\right)$ & $R_{\mathrm{out}}^{2}\left(I_{\sigma}\right)$ & $R_{\text {out }}^{2}\left(J_{\sigma}\right)$ & $R_{\text {out }}^{2}\left(\Sigma^{-1}\right)$ \\
\hline direct & $0.038 \pm 0.004$ & $0.732 \pm 0.006$ & $-0.311 \pm 0.004$ & $0.038 \pm 0.004$ & $0.732 \pm 0.006$ & $-0.293 \pm 0.004$ \\
\hline whitening & $-0.025 \pm 0.004$ & $-0.047 \pm 0.012$ & $-0.061 \pm 0.003$ & $-0.031 \pm 0.004$ & $-0.192 \pm 0.013$ & $-0.061 \pm 0.003$ \\
\hline whitening $\star$ & $0.059 \pm 0.004$ & $0.277 \pm 0.010$ & $-0.056 \pm 0.003$ & $0.047 \pm 0.004$ & $0.152 \pm 0.012$ & $-0.056 \pm 0.004$ \\
\hline el & $-0.631 \pm 0.010$ & $-1.770 \pm 0.038$ & $-0.262 \pm 0.005$ & $-0.642 \pm 0.010$ & $-1.785 \pm 0.038$ & $-0.260 \pm 0.005$ \\
\hline elᄎ & $-0.128 \pm 0.008$ & $0.727 \pm 0.005$ & $-0.369 \pm 0.008$ & $-0.133 \pm 0.008$ & $0.701 \pm 0.005$ & $-0.360 \pm 0.008$ \\
\hline kyle & $0.343 \pm 0.003$ & $0.822 \pm 0.003$ & $0.214 \pm 0.003$ & $0.336 \pm 0.003$ & $0.808 \pm 0.004$ & $0.211 \pm 0.003$ \\
\hline$r$-direct & $0.276 \pm 0.004$ & $0.480 \pm 0.010$ & $0.180 \pm 0.003$ & $0.274 \pm 0.004$ & $0.479 \pm 0.010$ & $0.180 \pm 0.003$ \\
\hline $\mathrm{ml}$ & $0.373 \pm 0.003$ & $0.829 \pm 0.003$ & $0.215 \pm 0.003$ & $0.358 \pm 0.003$ & $0.803 \pm 0.004$ & $0.208 \pm 0.003$ \\
\hline$r-e l$ & $0.257 \pm 0.003$ & $0.661 \pm 0.005$ & $0.126 \pm 0.004$ & $0.249 \pm 0.003$ & $0.644 \pm 0.005$ & $0.124 \pm 0.004$ \\
\hline$r-e l \star$ & $0.236 \pm 0.004$ & $0.753 \pm 0.004$ & $0.090 \pm 0.004$ & $0.227 \pm 0.004$ & $0.733 \pm 0.005$ & $0.089 \pm 0.004$ \\
\hline r-kyle & $0.239 \pm 0.004$ & $0.788 \pm 0.004$ & $0.082 \pm 0.004$ & $0.232 \pm 0.004$ & $0.776 \pm 0.004$ & $0.081 \pm 0.004$ \\
\hline
\end{tabular}

Table 5: In-sample and out-sample scores for stocks. 
non-starred counterparts.

\section{Influence of liquidity on scores}

An interesting feature of our stocks dataset is the heterogeneity in liquidity among different stocks. This allows to explore the influence of the liquidity of a given stock on the performance of different models. We show the results of this analysis in Figure 10. Consistently with the scores obtained Table 5, we find that overall, in score terms, ml $>k y l e>r-d i r e c t>r-e l$. As one may expect, the $r$-direct model fares better for very liquid stocks, as a larger fraction of variance can be explained by idiosyncratic trades. Surprisingly, the same holds for $\mathrm{ml}$ and kyle models. The $\mathrm{r}$-el model stands as an exception: it better explains price moves for stocks which are within the band of typical liquidity, between $\omega_{10 \%}$ and $\omega_{90 \%}$, than for very liquid stocks. However, this is natural: the $r$-el model assumes an aggregated pool of liquidity (along the modes of the covariance matrix). Thus, though this assumption is roughly justified for stocks close to the average liquidity, it is violated outside of this zone. On the other hand, the $\mathrm{ml}$ and kyle models have no such assumption and can thus better deal with very liquid or illiquid stocks. To further reinforce this point, for stocks of liquidity close to the average in our pool of stocks, the difference scores of the el and kyle models reach a minimum. This is consistent with the fact that in the approximation $\Omega \approx \omega_{50 \%}$, the two models coincide. Thus, violating self-stability (Axiom 13) may be relevant to explain price changes for all ranges of liquidity within our basket of instruments.

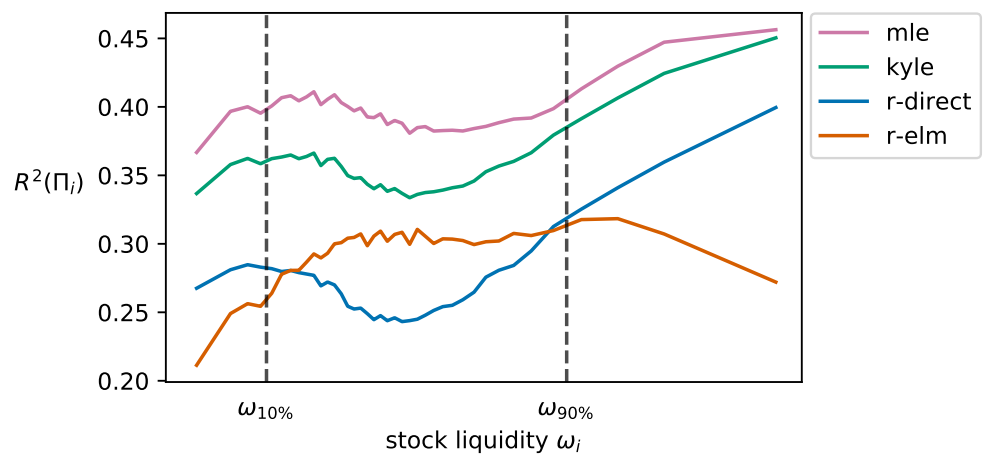

Figure 10: Idiosyncratic scores as a function of liquidity.

For each stock in our dataset, we compute the the in-sample stock-specific scores $R^{2}\left(\Pi_{i}\right)$ scores on 2016 data. We then represent the average in-sample stock-specific score as a function of the liquidity $\omega_{i}$, binning data by $\omega_{i}$ to smooth out noise. Results for the ml (in pink), kyle (in green), $r$-direct (in blue) and $r$-el (in orange) models are shown. We have further indicated the $10 \%$ and $90 \%$ quantiles of liquidity $\omega_{10 \%}$ and $\omega_{90 \%}$.

\subsection{Robustness}

The previous section compared the descriptive power of different cross-impact models. However, axioms also constrain cross-impact models and thus change the degrees of freedom of models. We are thus interested in investigating the robustness of cross-impact models on our datasets.

\subsubsection{Overfitting and non stationarity in predictive power}

Recall that we write $R_{X \rightarrow Y}^{2}(M)$ for the average score for an error matrix $M$. In-sample scores are defined as $R_{\text {in }}^{2}(M):=$ $\frac{1}{2}\left(R_{2016 \rightarrow 2016}^{2}(M)+R_{2017 \rightarrow 2017}^{2}(M)\right)$ while out-of-sample scores are defined as $R_{\text {out }}(M):=\frac{1}{2}\left(R_{2016 \rightarrow 2017}^{2}(M)+R_{2017 \rightarrow 2016}^{2}(M)\right)$. To study the non-stationarity in predictive power, we compute $\frac{\left|R_{2016}^{2}(M)-R_{2017}^{2}(M)\right|}{R_{2016}^{2}(M)+R_{2017}^{2}(M)}$, which we refer to as the difference in predictability. When this quantity is equal to zero, it signifies data from 2016 is as easily predictable as data from 2017 . A difference indicates that the predictive power of the model has changed from one year to the other. In Figure 11 we compare overfitting to predictability difference. Overall, overfitting is smaller than predictability difference, which shows that models are indeed robust across time and their performance is not dictated by changes in predictability of the underlying price changes. 


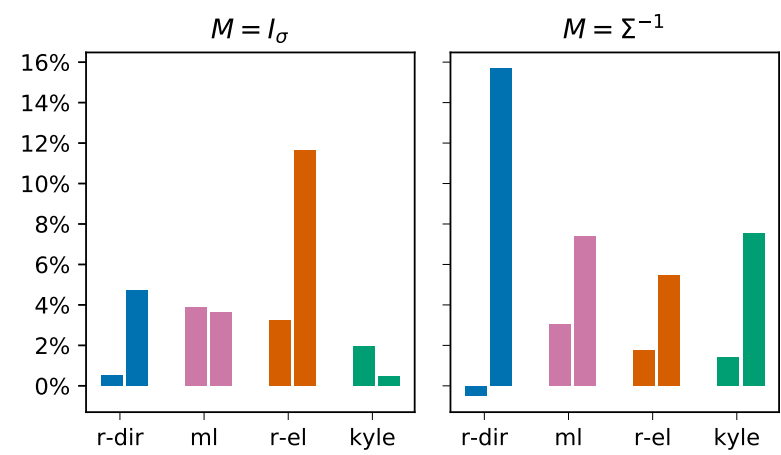

$\square$ overfitting

Figure 11: Overfitting and difference predictability scores for different models.

We show side by side the comparison of overfitting coefficient $1-\frac{R_{\mathrm{out}(M)}^{2}}{R_{\mathrm{in}(M)}^{2}}$ (with no shading) and non-stationarity in predictive power $\frac{\left|R_{2016}^{2}(M)-R_{2017}^{2}(M)\right|}{R_{2016}^{2}(M)+R_{2017}^{2}(M)}$ (with shading) for the $r$-direct (in blue), $\mathrm{ml}$ (in pink), $\mathrm{r}$-el (in orange) and kyle (in green) models for idiosyncratic $M=I_{\boldsymbol{\sigma}}$ (left) and risk-weighted $M=\Sigma^{-1}$ (right) scores.

\subsubsection{Influence of the number of instruments and of the timescale of study}

Across our asset classes, we have focused on the 1 minute timescale. Now, we examine which models better explain price changes on longer timescales. In Figure 12, we show the out-of-sample score and overfitting coefficient for idiosyncratic price changes for our set of 393 stocks, as a function of the bin timescale and number of instruments.
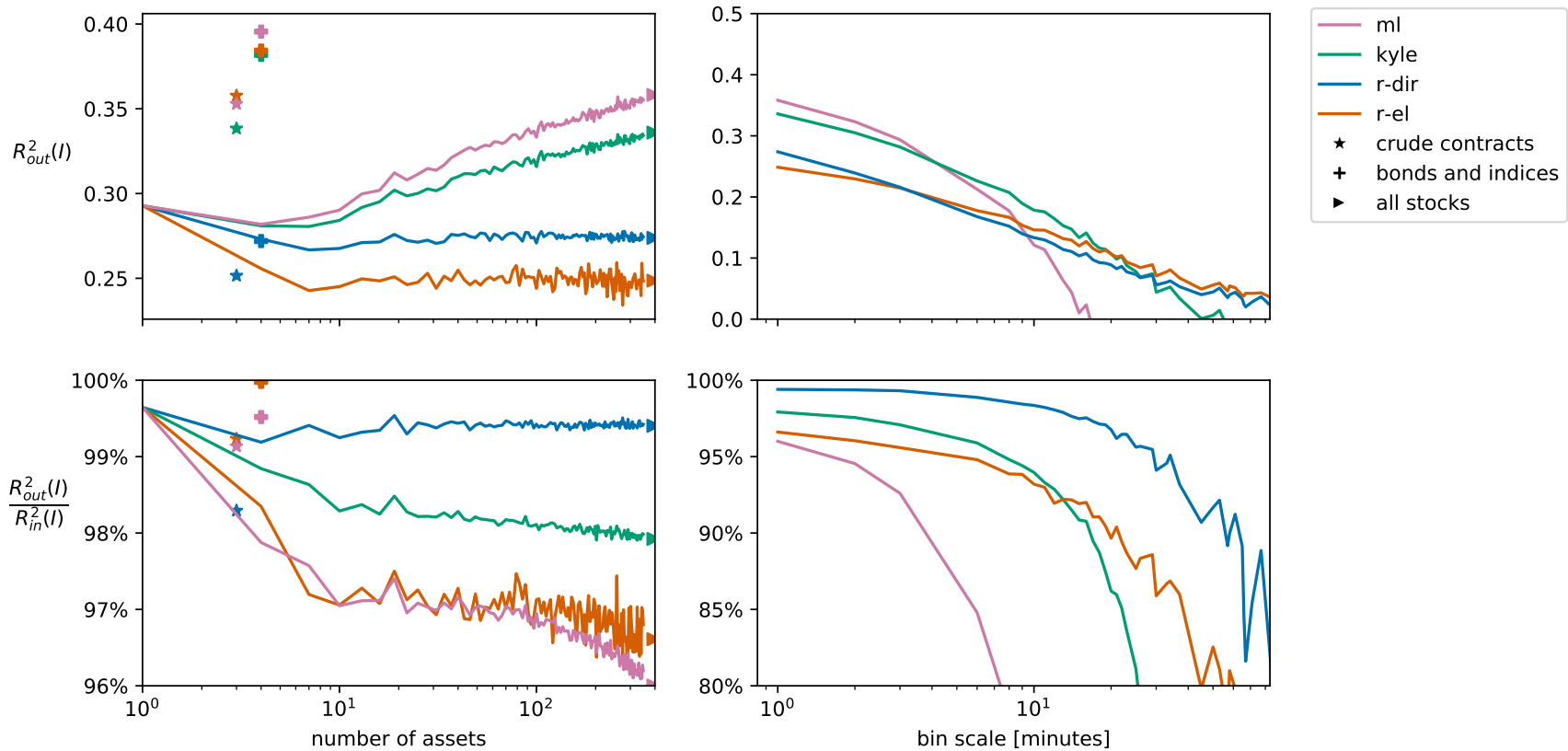

Figure 12: Idiosyncratic score and overfitting as a function of the number of assets and bin timescale.

Left column: average out-of-sample idiosyncratic score $R_{\text {out }}^{2}\left(I_{\boldsymbol{\sigma}}\right)$ (top left) and overfitting coefficient $\frac{R_{\text {out }}^{2}\left(I_{\boldsymbol{\sigma}}\right)}{R_{\mathrm{in}}^{2}\left(I_{\boldsymbol{\sigma}}\right)}$ (bottom left) computed using stocks data. Out-of-sample and in-sample scores were computed by randomly selecting a subset of stocks and computing scores on the given subset, repeating the procedure more when there are fewer stocks are selected than when a large proportion of stocks from our sample is considered. The average score for each models across all samples is then shown. Scores are shown for the $\mathrm{ml}$ (in pink), the kyle (in green), $r$-direct (in blue) and $r$-el (in orange). Stars show results for crude contracts, crosses for bonds and indices and triangles for all 393 stocks of our sample. Right column: idiosyncratic scores (top right) and overfitting coefficient (bottom right) as a function of the bin timescale. Scores were computed using the same procedure described in Section 4.4, varying the bin parameter from 10 seconds up to around an hour. 
As expected, the number of degrees of freedom controls the overfitting of different models. This explains why, in terms of overfitting with respect to the number of instruments at the minute timescale, $r$-direct $<\mathrm{kyle}<\mathrm{ml} \approx \mathrm{r}$-el. In contrast, models overfit less on futures, which suggests that overfitting decreases as the pairwise correlation between instruments increases. Furthermore, out-of-sample idiosyncratic scores for the $\mathrm{ml}$ and kyle model increase with the number of assets. A somewhat surprising result, despite the small pairwise correlation of instruments in our stock dataset and the large number of stocks considered in this study, is that idiosyncratic scores appear to keep increasing for more than 400 assets. Nevertheless, cross-impact models score much better for futures than for stocks, suggesting that scores also increase as the pairwise correlation between instruments increases.

Focusing on to the influence of the bin timescale, there is little overfitting at the minute timescale but it increases with the bin timescale. In particular, the good fit of the $\mathrm{ml}$ at small timescales quickly breaks down for larger timescales. On the other hand, both the $r-e l$ and kyle models are quite robust up until the 10 minute timescale. This highlights the importance of enforcing consistency requirements to reduce overfitting.

\section{Conclusion}

Let us summarise what we have achieved. Our main object was to build a series of theoretical prescriptions to choose the most suitable cross-impact model $\Lambda$ given a set of empirical observations of market data. To do so, we generically defined a cross-impact model as a function of second-order statistics, constrained through a certain number of axioms. From a theoretical standpoint, no matter the specific framework for building cross-impact models, one can easilly understand that proceeding in this fashion is very useful as it significantly reduces the universe of possible models. Empirical evidence confirms the importance of fragmentation invariance axioms (Axioms 8 to 10) for cross-impact models applied to markets where some instruments (or linear combination of instruments) display very small fluctuations. Stability axioms enable models to better explain price moves of instruments in extreme liquidity/illiquidity conditions (see Figure 10). Additionally, the reduced number of parameters of models constrained by axioms (such as the kyle model) reduces overfitting - both with the number of instruments in our tradeable universe and with the timescale of study (see Figure 12). In all markets studied, our analysis confirms that cross-impact models are well suited to predict execution costs and evaluate liquidity risk, showing significant improvement compared to impact models which ignore cross-sectional effects (see Tables 2, 3 and 5).

Though our framework focused on the linear static scenario, the prescriptions and models introduced in this paper could be generalised to deal with more general cases. Furthermore, the framework can be adapted to deal with derivatives, which we leave as the topic of future work. Another topic is the generalisation of this framework to account for the auto-correlation of the order flow. In [18], the authors began to study this question, paving the way to a cross-impact generalisation of [12]. Finally, many questions subsist about the underlying dynamics or supply and demand which account for cross-impact: from the microstructural perspective what coupling of order-book dynamics could account for such aggregate price dynamics? This question requires further attention and we hope to examine it in detail from the perspective of zero-intelligence limit order-book models (see e.g. [15]).

\section{Acknowledgments}

We warmly thank J.-P. Bouchaud, Z. Eisler and B. Toth, M. Rosenbaum and A. Fosset for fruitful discussions. This research was conducted within the Econophysics \& Complex Systems Research Chair under the aegis of the Fondation du Risque, a joint initiative by the Fondation de l'École polytechnique, l'École polytechnique and Capital Fund Management. 


\section{References}

[1] Aurélien Alfonsi, Florian Klöck, and Alexander Schied. Multivariate transient price impact and matrix-valued positive definite functions. Mathematics of operations research, 41(3):914-934, 2016.

[2] Robert Almgren, Chee Thum, Emmanuel Hauptmann, and Hong Li. Direct estimation of equity market impact. Risk, 18(7):58-62, 2005.

[3] Michael Benzaquen, Iacopo Mastromatteo, Zoltan Eisler, and Jean-Philippe Bouchaud. Dissecting cross-impact on stock markets: An empirical analysis. Journal of Statistical Mechanics: Theory and Experiment, 2017(2):23406, 2017.

[4] Jean-Philippe Bouchaud, Julius Bonart, Jonathan Donier, and Martin Gould. Trades, Quotes and Prices. Cambridge University Press, 32018.

[5] Jordi Caballe and Murugappa Krishnan. Imperfect competition in a multi-security market with risk neutrality. Econometrica (1986-1998), 62(3):695, 1994.

[6] Pierre Cardaliaguet and Charles-Albert Lehalle. Mean field game of controls and an application to trade crowding. Mathematics and Financial Economics, 12(3):335-363, 2018.

[7] Luis Carlos del Molino, Iacopo Mastromatteo, Michael Benzaquen, and Jean-Philippe Bouchaud. The Multivariate Kyle model: More is different. 2018.

[8] David Evangelista and Douglas Vieira. New closed-form approximations in multi-asset market making. arXiv preprint arXiv:1810.04383, 2018.

[9] Jim Gatheral. No-Dynamic-Arbitrage and Market Impact. Technical report, 2009.

[10] Olivier Guéant. Optimal market making. Applied Mathematical Finance, 24(2):112-154, 2017.

[11] Joel Hasbrouck and Duane J Seppi. Common factors in prices, order flows, and liquidity. Journal offinancial Economics, 59(3):383-411, 2001.

[12] Thibault Jaisson. Market impact as anticipation of the order flow imbalance. Quantitative Finance, 15(7):1123-1135, 2015.

[13] Charles-Albert Lehalle and Charafeddine Mouzouni. A Mean Field Game of Portfolio Trading and Its Consequences On Perceived Correlations. arXiv preprint arXiv:1902.09606, 2019.

[14] Iacopo Mastromatteo, Michael Benzaquen, Zoltan Eisler, and Jean-Philippe Bouchaud. Trading lightly: Cross-impact and optimal portfolio execution. 2017.

[15] Iacopo Mastromatteo, Bence Toth, and Jean-Philippe Bouchaud. Agent-based models for latent liquidity and concave price impact. Physical Review E, 89(4):042805, 2014.

[16] Paolo Pasquariello and Clara Vega. Strategic cross-trading in the us stock market. Review of Finance, 19(1):229-282, 2015.

[17] Michael Schneider, Fabrizio Lillo, Michael Benzaquen, Jean-Philippe Bouchaud, Katia Colaneri, Thomas Guhr, Florian Klöck, Enrico Melchioni, Loriana Pelizzon, and Damian Taranto. Cross-impact and no-dynamic-arbitrage. Technical report, 2017.

[18] Mehdi Tomas and Mathieu Rosenbaum. From microscopic price dynamics to multidimensional rough volatility models. arXiv preprint arXiv:1910.13338, 2019.

[19] Nicolo Torre. BARRA market Impact model handbook. BARRA Inc., Berkeley, 1997.

[20] Shanshan Wang, Sebastian Neusüß, and Thomas Guhr. Grasping asymmetric information in market impacts. arXiv preprint arXiv:1710.07959, 2017.

[21] Shanshan Wang, Rudi Schäfer, and Thomas Guhr. Price response in correlated financial markets: empirical results. arXiv preprint arXiv:1510.03205, 2015.

[22] Shanshan Wang, Rudi Schäfer, and Thomas Guhr. Cross-response in correlated financial markets: individual stocks. The European Physical Journal B, 89(4):105, 2016. 


\section{A Models and Axioms}

This section proves some of the results stated in Table 1 and implications between the different axioms.

\section{A.1 Relation between fragmentation and liquidity axioms}

Let us start us with a powerful lemma that allows to translate liquidity properties into fragmentation properties through split invariance.

Lemma 1. Let us consider a cross-impact model $\Lambda$ that is split-invariant and a subspace $V$ such that $\varnothing \subset V \subseteq \mathbb{R}^{n}$. Define the regularized orderflow-covariance and response matrices:

$$
\begin{aligned}
\Omega^{\prime} & =\left(\bar{\Pi}_{V}+\epsilon \Pi_{V}\right) \Omega\left(\bar{\Pi}_{V}+\epsilon \Pi_{V}\right) \\
R^{\prime} & =R\left(\bar{\Pi}_{V}+\epsilon \Pi_{V}\right) .
\end{aligned}
$$

Then we have:

$$
\begin{aligned}
\Lambda\left(\Sigma, \Omega^{\prime}, R^{\prime}\right) & =\bar{\Pi}_{V} \Lambda\left(\Sigma^{\prime \prime}, \Omega, R^{\prime \prime}\right) \bar{\Pi}_{V} \\
& +\epsilon^{-1} \bar{\Pi}_{V} \Lambda\left(\Sigma^{\prime \prime}, \Omega, R^{\prime \prime}\right) \Pi_{V} \\
& +\epsilon^{-1} \Pi_{V} \Lambda\left(\Sigma^{\prime \prime}, \Omega, R^{\prime \prime}\right) \bar{\Pi}_{V} \\
& +\epsilon^{-2} \Pi_{V} \Lambda\left(\Sigma^{\prime \prime}, \Omega, R^{\prime \prime}\right) \Pi_{V}
\end{aligned}
$$

where

$$
\begin{aligned}
& \Sigma^{\prime \prime}=\left(\bar{\Pi}_{V}+\epsilon \Pi_{V}\right) \Sigma\left(\bar{\Pi}_{V}+\epsilon \Pi_{V}\right) \\
& R^{\prime \prime}=\left(\bar{\Pi}_{V}+\epsilon \Pi_{V}\right) R
\end{aligned}
$$

Proof. By split invariance, we can write:

$$
\Lambda\left(\Sigma, \Omega^{\prime}, R^{\prime}\right)=D \Lambda\left(D^{-1} \Sigma D^{-1}, D \Omega^{\prime} D, D^{-1} R^{\prime} D\right) D
$$

where we choose $D=\left(\bar{\Pi}_{V}+\epsilon^{-1} \Pi_{V}\right)$. We can always do this with $D$ diagonal as long as $V$ is generated by the canonical basis. We then obtain:

$$
\Lambda\left(\Sigma, \Omega^{\prime}, R^{\prime}\right)=\left(\bar{\Pi}_{V}+\epsilon^{-1} \Pi_{V}\right) \Lambda\left(D^{-1} \Sigma D^{-1}, \Omega, D^{-1} R\right)\left(\bar{\Pi}_{V}+\epsilon^{-1} \Pi_{V}\right),
$$

which, upon substitution of $D^{-1}$ yields the result.

This implies that, if one assumes fragmentation invariance and controls the speed of divergence of the remaining terms, one can show cross-stability results.

Proposition 5. Let a cross-impact model $\Lambda$ satisfy split symmetry (Axiom 4), semi-strong fragmentation invariance (Axiom 9). Then:

(i) $\Lambda$ is weakly cross-stable (Axiom 11) if

$$
\epsilon^{-1} \bar{\Pi}_{V} \Lambda\left(\Sigma^{\prime \prime}, \Omega, R^{\prime \prime}\right) \Pi_{V} \underset{\varepsilon \rightarrow 0}{=} O(1) .
$$

(ii) If, additionally, $\Lambda$ is continuous in the first and third argument strongly fragmentation invariant, then $\Lambda$ is strongly cross-stable (Axiom 12).

(iii) $\Lambda$ is self-stable (Axiom 13) if

$$
\epsilon^{-2} \Pi_{V} \Lambda\left(\Sigma^{\prime \prime}, \Omega, R^{\prime \prime}\right) \Pi_{V} \underset{\varepsilon \rightarrow 0}{=} O(1) .
$$

Proof. Using the results of Lemma 1, if we assume that

$$
\epsilon^{-1} \bar{\Pi}_{V} \Lambda\left(\Sigma^{\prime \prime}, \Omega, R^{\prime \prime}\right) \Pi_{V} \underset{\varepsilon \rightarrow 0}{=} O(1),
$$

then $\Lambda$ is weakly cross-stable (Axiom 11). Further assuming continuity at $\epsilon=0$ and strong fragmentation invariance, we obtain

$$
\bar{\Pi}_{V} \Lambda\left(\Sigma, \Omega^{\prime}, R^{\prime}\right) \bar{\Pi}_{V}=\bar{\Pi}_{V} \Lambda\left(\Sigma^{\prime \prime}, \Omega, R^{\prime \prime}\right) \bar{\Pi}_{V} \underset{\varepsilon \rightarrow 0}{=} \bar{\Pi}_{V} \Lambda\left(\Sigma^{\prime \prime}, \Omega, R^{\prime \prime}\right) \bar{\Pi}_{V}+o(1)
$$

Strong fragmentation invariance (Axiom 10) thus implies strong cross-stability (Axiom 12). Finally, if

$$
\epsilon^{-2} \Pi_{V} \Lambda\left(\Sigma^{\prime \prime}, \Omega, R^{\prime \prime}\right) \Pi_{V} \underset{\varepsilon \rightarrow 0}{=} O(1)
$$

then Lemma 1 implies that $\Lambda$ is self-stable. 
One can also exploit the same lemma and try to establish a link with the fragmentation invariance properties. In a similar fashion as Lemma 1, one can prove the following Lemma.

Lemma 2. Let us consider a cross-impact model $\Lambda$ that is split-invariant and a subspace $V$ such that $\varnothing \subset V \subseteq \mathbb{R}^{n}$, Define the regularized price-covariance and response matrices:

$$
\begin{aligned}
& \Sigma^{\prime}:=\left(\bar{\Pi}_{V}+\epsilon \Pi_{V}\right) \Sigma\left(\bar{\Pi}_{V}+\epsilon \Pi_{V}\right) \\
& R^{\prime}:=\left(\bar{\Pi}_{V}+\epsilon \Pi_{V}\right) R .
\end{aligned}
$$

Then we have:

$$
\begin{aligned}
\Lambda\left(\Sigma^{\prime}, \Omega, R^{\prime}\right) & =\bar{\Pi}_{V} \Lambda\left(\Sigma, \Omega^{\prime \prime}, R^{\prime \prime}\right) \bar{\Pi}_{V} \\
& +\epsilon^{-1} \bar{\Pi}_{V} \Lambda\left(\Sigma, \Omega^{\prime \prime}, R^{\prime \prime}\right) \Pi_{V} \\
& +\epsilon^{-1} \Pi_{V} \Lambda\left(\Sigma, \Omega^{\prime \prime} R^{\prime \prime}\right) \bar{\Pi}_{V} \\
& +\epsilon^{-2} \Pi_{V} \Lambda\left(\Sigma, \Omega^{\prime \prime}, R^{\prime \prime}\right) \Pi_{V}
\end{aligned}
$$

where:

$$
\begin{aligned}
\Omega^{\prime \prime} & :=\left(\bar{\Pi}_{V}+\epsilon \Pi_{V}\right) \Omega\left(\bar{\Pi}_{V}+\epsilon \Pi_{V}\right) \\
R^{\prime \prime} & :=R\left(\bar{\Pi}_{V}+\epsilon \Pi_{V}\right) .
\end{aligned}
$$

Proposition 6. Let $\Lambda$ be a split-invariant, weakly cross-stable and self-stable cross-impact model (Axioms 4, 11 and 13). Then if $\operatorname{ker} \Sigma$ can be generated by the canonical basis, then $\Lambda$ is semi-strongly fragmentation invariant (Axiom 9).

Proof. Let us assume that it is possible to generate $\operatorname{ker}(\Sigma)$ with the physical (canonical) basis. In that case, one can choose $V=\operatorname{ker}(\Sigma)$ and observe that, using the notations introduced in Lemma $2, \Sigma^{\prime}=\bar{\Pi}_{V} \Sigma \bar{\Pi}_{V}, R^{\prime}=\bar{\Pi}_{V} R$. Weak-cross stability (Axiom 11) implies:

$$
\begin{aligned}
& \bar{\Pi}_{V} \Lambda\left(\Sigma^{\prime}, \Omega^{\prime}, R^{\prime}\right) \Pi_{V} \underset{\epsilon \rightarrow 0}{=} O(1) \\
& \Pi_{V} \Lambda\left(\Sigma^{\prime}, \Omega^{\prime}, R^{\prime}\right) \bar{\Pi}_{V} \underset{\epsilon \rightarrow 0}{=} O(1) .
\end{aligned}
$$

Therefore, applying Lemma 2 yields

$$
\bar{\Pi}_{V} \Lambda\left(\Sigma^{\prime}, \Omega, R^{\prime}\right) \Pi_{V}=\epsilon^{-1} \bar{\Pi}_{V} \Lambda\left(\Sigma, \Omega^{\prime \prime}, R^{\prime \prime}\right) \Pi_{V} \underset{\epsilon \rightarrow 0}{=} O(1)
$$

and we obtain $\bar{\Pi}_{V} \Lambda\left(\Sigma, \Omega^{\prime \prime}, R^{\prime \prime}\right) \Pi_{V}=\bar{\Pi}_{V} \Lambda(\Sigma, \Omega, R) \Pi_{V} \underset{\epsilon \rightarrow 0}{\rightarrow} 0$. (similarly, $\left.\bar{\Pi}_{V} \Lambda(\Sigma, \Omega, R) \Pi_{V}=0\right)$. On the other hand, from self-stability (Axiom 13), we obtain $\Pi_{V} \Lambda(\Sigma, \Omega, R) \Pi_{V} \underset{\epsilon \rightarrow 0}{\rightarrow} 0$. Combined, we thus have proved Axioms 8 and 9 .

\section{A.2 Proof of Proposition 3}

Proposition 7. Let $\Lambda$ be a symmetric, positive-definite and price-covariance consistent cross-impact model (Axioms 6 , 7 and 14). Then $\Lambda=\Lambda_{\mathrm{kyle}}$ up to a multiplicative constant.

Proof. Let $\Lambda$ be a cross-impact model which satisfies Axioms 6 and 14 and $(\Sigma, \Omega, R) \in\left(\mathscr{S}_{n}^{+} \times \mathscr{S}_{n}^{++} \times \mathscr{M}_{n}\right)$. Then, we have, writing $\Lambda$ for $\Lambda(\Sigma, \Omega, R)$, and $\mathscr{L}$ a matrix such that $\Omega=\mathscr{L}^{\top}$,

$$
\Sigma=\Lambda \Omega \Lambda^{\top}=\Lambda \mathscr{L} \mathscr{L}^{\top} \Lambda^{\top}=(\Lambda \mathscr{L})(\Lambda \mathscr{L})^{\top}
$$

Thus, by unicity up to a rotation of the square root decomposition, writing $\mathscr{G}$ for a matrix such that $\Sigma=\mathscr{G} \mathscr{G}^{\top}$, there exists $O$ such that $\Lambda=\mathscr{G} O \mathscr{L}^{-1}$. Furthermore, since $\Lambda$ is symmetric,

$$
\mathscr{G} O \mathscr{L}^{-1}=\left(\mathscr{G} O \mathscr{L}^{-1}\right)^{T} .
$$

Rewriting, we find

$$
O^{T}=\mathscr{L}^{T} \mathscr{G} O \mathscr{L}^{-1} \mathscr{G}^{-T}
$$


so that

$$
\begin{aligned}
\mathbb{\nabla} & =O \mathscr{L}^{\top} \mathscr{G} O \mathscr{L}^{-1} \mathscr{G}^{-\top} \\
& =O\left(\mathscr{L}^{\top} \mathscr{G}\right) O\left(\mathscr{L}^{\top} \mathscr{G}\right)^{-\top} \\
& =O\left(\mathscr{L}^{\top} \mathscr{G}\right) O\left(\mathscr{L}^{\top} \mathscr{G}\right)\left(\mathscr{L}^{\top} \mathscr{G}\right)^{-1}\left(\mathscr{L}^{\top} \mathscr{G}\right)^{-\top} .
\end{aligned}
$$

Finally, we have:

$$
\left(O \mathscr{L}^{\top} \mathscr{G}\right)^{2}=\left(\mathscr{L}^{\top} \mathscr{G}\right)^{\top}\left(\mathscr{L}^{\top} \mathscr{G}\right) .
$$

Since $\left(\mathscr{L}^{\top} \mathscr{G}\right)^{\top}\left(\mathscr{L}^{\top} \mathscr{G}\right)$ is symmetric positive semi-definite, the square root is unique and

$$
O \mathscr{L}^{T} \mathscr{G}=\sqrt{\left(\mathscr{L}^{T} \mathscr{G}\right)^{T}\left(\mathscr{L}^{T} \mathscr{G}\right)}
$$

which concludes the proof.

\section{A.3 Proof of Proposition 4}

Let us start with a lemma that will be used throughout this subsection.

Lemma 3. Let $\Lambda$ be a cross-impact model which satisfies Axioms 4 and 5 . Then, for all $(\Sigma, \Omega, R) \in\left(\mathscr{S}_{n}^{+} \times \mathscr{S}_{n}^{++} \times \mathscr{M}_{n}\right)$, it can be written as

$$
\Lambda(\Sigma, \Omega, R)=\mathscr{L}^{-\top} U \Lambda\left(U^{\top} \hat{\Sigma} U, \llbracket, U^{\top} \hat{R} U\right) U^{\top} \mathscr{L}^{-1},
$$

where

$$
\begin{aligned}
& \Omega=\mathscr{L} \mathscr{L}^{\top} \\
& \hat{\Sigma}=\mathscr{L}^{\top} \Sigma \mathscr{L} \\
& \hat{R}=\mathscr{L}^{\top} R \mathscr{L}^{-\top}
\end{aligned}
$$

and $U$ is an orthogonal matrix (i.e., $U U^{\top}=\rrbracket$ ).

Proof. The lemma is obtained by applying sequentially rotational invariance, split invariance and again rotational invariance. The first two transformations can be used in order to remove the dependency in $\Omega$ as the second argument of the $\Lambda(\Sigma, \Omega, R)$ function.

Proposition 8. A return covariance based cross-impact model $\Lambda$ that is both split invariant and rotational invariant (Axioms 4 and 5) can always be written in the form

$$
\Lambda(\Sigma, \Omega)=\mathscr{L}^{-\top} U F(\boldsymbol{\mu}) U^{\top} \mathscr{L}^{-1},
$$

where

$$
\Omega=\mathscr{L}^{\top} \quad ; \quad \hat{\Sigma}:=\mathscr{L}^{\top} \Sigma \mathscr{L} \quad ; \quad U^{\top} \hat{\Sigma} U:=\operatorname{diag}(\boldsymbol{\mu}) \quad ; \quad F(\boldsymbol{\mu}):=\Lambda(\operatorname{diag}(\boldsymbol{\mu}), \mathbb{1}) .
$$

Furthermore, if $\Lambda$ is cash-invariant and direct-invariant Axioms 2 and 3 , then $F(\boldsymbol{\mu}) \propto \operatorname{diag}(\boldsymbol{\mu})^{1 / 2}$ and $\Lambda=\Lambda_{\mathrm{kyle}}$ up to a multiplicative constant.

Proof. For a return covariance based model, we can simply choose from Eq. (3) to fix $U$ as the rotation that diagonalizes the symmetric matrix $\hat{\Sigma}$, obtaining:

$$
U^{\top} \hat{\Sigma} U=\operatorname{diag}(\boldsymbol{\mu}) .
$$

This choice implies:

$$
\Lambda(\Sigma, \Omega)=\mathscr{L}^{-\top} U \Lambda\left(\operatorname{diag}(\boldsymbol{\mu}), \text { 凹) } U^{\top} \mathscr{L}^{-1},\right.
$$

which yields the result of the first part of the proposition. Furthermore, if we assume $\Lambda$ is cash-invariant and direct-invariant Axioms 2 and 3 ,

$$
\Lambda(\operatorname{diag}(\boldsymbol{\mu}), \mathbb{\square})=\sum_{i=1}^{d} \sqrt{\mu_{i}} \Lambda\left(\boldsymbol{e}_{i} \boldsymbol{e}_{\boldsymbol{i}}^{\top}, \boldsymbol{e}_{i} \boldsymbol{e}_{\boldsymbol{i}}^{\top}\right)
$$

which yields the kyle model up to a constant. 


\section{A.4 Proof of some properties of the kyle model}

Lemma 4. The kyle model is strongly cross-stable in the sense of Axioms 12 and 13 and is not self-stable in the sense of Axiom 13.

Proof. Let $V$ be a linear subspace of $\mathbb{R}^{n}$ ) and $\varepsilon>0$. Note that, writing $\mathscr{G}$ for a matrix such that $\mathscr{G} \mathscr{G}^{\top}=\Sigma$, for any matrix $\mathscr{L}_{\varepsilon}$ such that $\mathscr{L}_{\varepsilon} \mathscr{L}_{\varepsilon}^{\top}=\Omega_{\varepsilon}$, there exists a rotation matrix $O_{\varepsilon}$ such that we have

$$
\Lambda_{\mathrm{kyle}}=\mathscr{G} O_{\varepsilon} \mathscr{L}_{\varepsilon}^{-1} \text {. }
$$

However, $\Omega_{\varepsilon}=\left(\bar{\Pi}_{V}+\varepsilon \Pi_{V}\right) \Omega\left(\bar{\Pi}_{V}+\varepsilon \Pi_{V}\right)=\left(\bar{\Pi}_{V}+\varepsilon \Pi_{V}\right) \mathscr{L}^{\top}\left(\bar{\Pi}_{V}+\varepsilon \Pi_{V}\right)=\left[\left(\bar{\Pi}_{V}+\varepsilon \Pi_{V}\right) \mathscr{L}\right]\left[\left(\bar{\Pi}_{V}+\varepsilon \Pi_{V}\right) \mathscr{L}\right]^{\top}$. Thus,

$$
\begin{aligned}
\Lambda_{\mathrm{kyle}} & =\mathscr{G} O_{\varepsilon}\left[\left(\bar{\Pi}_{V}+\varepsilon \Pi_{V}\right) \mathscr{L}\right]^{-1} \\
& =\mathscr{G} O_{\varepsilon} \mathscr{L}^{-1}\left(\bar{\Pi}_{V}+\frac{1}{\varepsilon} \Pi_{V}\right) \\
& =\mathscr{G O} O_{\varepsilon} \mathscr{L}^{-1} \bar{\Pi}_{V}+\frac{1}{\varepsilon} \mathscr{G} O_{\varepsilon} \mathscr{L}^{-1} \Pi_{V} .
\end{aligned}
$$

Using the symmetry of the kyle model, the above yields:

$$
\Lambda_{\mathrm{kyle}}=\bar{\Pi}_{V} \mathscr{L}^{-\top} O_{\varepsilon}^{\top} \mathscr{G}^{\top}+\frac{1}{\varepsilon} \Pi_{V} \mathscr{L}^{-\top} O_{\varepsilon}^{\top} \mathscr{G}^{\top} .
$$

Thus, we have:

$$
\bar{\Pi}_{V} \Lambda_{\mathrm{kyle}} \Pi_{V}=\bar{\Pi}_{V} \mathscr{L}^{-\top} O_{\varepsilon}^{\top} \mathscr{G}^{\top} \Pi_{V},
$$

and, as $O_{\varepsilon}^{\top}$ is an orthogonal matrix:

$$
\epsilon^{\gamma} \bar{\Pi}_{V} \Lambda_{\mathrm{kyle}} \Pi_{V} \underset{\varepsilon \rightarrow 0}{=} O(1)
$$

which proves Axiom 11 weak-cross stability. Furthermore,

$$
\Pi_{V} \Lambda_{\mathrm{kyle}} \Pi_{V}=\frac{1}{\varepsilon} \Pi_{V} \mathscr{L}^{-\top} O_{\varepsilon}^{\top} \mathscr{G}^{\top} \Pi_{V}
$$

so that unless $\Pi_{V} \mathscr{L}^{-\top} O_{\varepsilon}^{\top} \mathscr{G}^{\top} \Pi_{V}=0$, we have:

$$
\left\|\Pi_{V} \Lambda_{\mathrm{kyle}} \Pi_{V}\right\|=\varepsilon^{-1}\left\|\Pi_{V} \mathscr{L}^{-\top} O_{\varepsilon}^{\top} \mathscr{G}^{\top} \Pi_{V}\right\| \underset{\varepsilon \rightarrow 0}{\rightarrow} \infty .
$$

Choosing diagonal $\Sigma$ and $\Omega$ such that $\Pi_{V} \mathscr{L} \neq 0$ and $\mathscr{G} \Pi_{V} \neq 0$, we see that $\Pi_{V} \mathscr{L}^{-\top} O_{\varepsilon}^{\top} \mathscr{G}^{\top} \Pi_{V}=0$ cannot hold for all $\Sigma, \Omega$. This shows that kyle does not satisfy Axiom 13. Finally, notice that by using Lemma 3 one can make $\Omega$ appear only in the combination $\mathscr{L}^{\top} \Sigma \mathscr{L}$, which is insensitive to the components of $\Omega$ belonging to the kernel of $\Sigma$, which proves strong cross-stability.

\section{B Proofs of illustrative examples}

This section is devoted to proving the results given in Section 4, for completeness.

\section{B.1 Proof of Example 1}

We have:

$$
\Sigma=\left(\begin{array}{cc}
1 & \rho \sigma \\
\rho \sigma & \sigma^{2}
\end{array}\right)=\left(\begin{array}{cc}
1 & 0 \\
\sigma \rho & \sigma \sqrt{1-\rho^{2}}
\end{array}\right)\left(\begin{array}{cc}
1 & \sigma \rho \\
0 & \sigma \sqrt{1-\rho^{2}}
\end{array}\right)=\mathscr{G} \mathscr{G}^{T} .
$$

Thus, as we can express $O$ in the orthogonal group solely as a function of an angle $\theta$ as $O=\left(\begin{array}{cc}\cos \theta & -\sin \theta \\ \sin \theta & \cos \theta\end{array}\right)$, we can express the Kyle estimator as:

$$
\begin{aligned}
& \Lambda_{\mathrm{kyle}}=\mathscr{G} O \mathscr{L}^{-1}=\left(\begin{array}{cc}
1 & 0 \\
\sigma \rho & \sigma \sqrt{1-\rho^{2}}
\end{array}\right)\left(\begin{array}{cc}
\cos \theta & -\sin \theta \\
\sin \theta & \cos \theta
\end{array}\right)\left(\begin{array}{cc}
1 / \omega_{1} & 0 \\
0 & 1 / \omega_{2}
\end{array}\right) \\
& =\left(\begin{array}{cc}
\cos \theta / \omega_{1} & -\sin \theta / \omega_{2} \\
\frac{\sigma}{\omega_{1}}\left(\rho \cos \theta+\sqrt{1-\rho^{2}} \sin \theta\right) & \frac{\sigma}{\omega_{2}}\left(-\rho \sin \theta+\sqrt{1-\rho^{2}} \cos \theta\right)
\end{array}\right) \\
& =\frac{\cos \theta}{\omega_{1}}\left(\begin{array}{cc}
1 & -\frac{\omega_{1}}{\omega_{2}} \tan \theta \\
\sigma\left(\rho+\sqrt{1-\rho^{2}} \tan \theta\right) & \frac{\sigma \omega_{1}}{\omega_{2}}\left(-\rho \tan \theta+\sqrt{1-\rho^{2}}\right)
\end{array}\right)
\end{aligned}
$$


Imposing the symmetry yields $\theta=-\arctan \frac{\sigma \rho}{\frac{\omega_{1}}{\omega_{2}}+\sigma \sqrt{1-\rho^{2}}}$. Injecting in the above we have:

$$
\begin{aligned}
\Lambda_{\mathrm{kyle}} & =\frac{\cos \theta}{\omega_{1}}\left(\begin{array}{cc}
1 & -\frac{\omega_{1}}{\omega_{2}} \tan \theta \\
\sigma\left(\rho+\sqrt{1-\rho^{2}} \tan \theta\right) & \frac{\sigma \omega_{1}}{\omega_{2}}\left(-\rho \tan \theta+\sqrt{1-\rho^{2}}\right)
\end{array}\right) \\
& =\frac{\cos \theta}{\omega_{1}}\left(\begin{array}{cc}
\frac{\sigma \rho}{1+\frac{\omega_{2}}{\omega_{1}} \sigma \sqrt{1-\rho^{2}}} \\
\frac{\sigma \rho}{1+\frac{\omega_{2}}{\omega_{1}} \sigma \sqrt{1-\rho^{2}}} & \frac{\sigma \omega_{1}}{\omega_{2}} \sqrt{1-\rho^{2}}-\frac{\sigma \omega_{1}}{\omega_{2}} \rho \tan \theta
\end{array}\right) \\
& =\frac{1}{\omega_{2}} \frac{1}{\sqrt{\frac{\omega_{1}^{2}}{\omega_{2}^{2}}+2 \frac{\omega_{1}}{\omega_{2}} \sigma \sqrt{1-\rho^{2}}+\sigma^{2}}}\left(\begin{array}{cc}
1+\frac{\omega_{2}}{\omega_{1}} \sigma \sqrt{1-\rho^{2}} & \left(1+\frac{\omega_{2}}{\omega_{1}} \sigma \sqrt{1-\rho^{2}}\right)\left(\frac{\sigma \omega_{1}}{\omega_{2}} \sqrt{1-\rho^{2}}-\frac{\sigma \omega_{1}}{\omega_{2}} \rho \tan \theta\right)
\end{array}\right) \\
& =\frac{1}{\sqrt{\omega_{1}^{2}+2 \omega_{1} \omega_{2} \sigma \sqrt{1-\rho^{2}}+\omega_{1}^{2} \sigma^{2}}}\left(\begin{array}{cc}
1+\frac{\omega_{2}}{\omega_{1}} \sigma \sqrt{1-\rho^{2}} & \sigma \rho \\
\sigma \rho & \left(1+\frac{\omega_{2}}{\omega_{1}} \sigma \sqrt{1-\rho^{2}}\right)\left(\frac{\sigma \omega_{1}}{\omega_{2}} \sqrt{1-\rho^{2}}-\frac{\sigma \omega_{1}}{\omega_{2}} \rho \tan \theta\right)
\end{array}\right) \\
& =\frac{\omega_{2}}{\sqrt{\omega_{1}^{2}+2 \omega_{1} \omega_{2} \sigma \sqrt{1-\rho^{2}}+\omega_{1}^{2} \sigma^{2}}}\left(\begin{array}{c}
1+\frac{\omega_{2}}{\omega_{1}} \sigma \sqrt{1-\rho^{2}} \\
\sigma \rho
\end{array}\right)
\end{aligned}
$$

Finally,

$$
\Lambda_{\mathrm{kyle}}=\frac{1}{\sqrt{\omega_{1}^{2}+2 \omega_{1} \omega_{2} \sigma \sqrt{1-\rho^{2}}+\omega_{2}^{2} \sigma^{2}}}\left(\begin{array}{cc}
1+\frac{\omega_{2}}{\omega_{1}} \sigma \sqrt{1-\rho^{2}} & \sigma \rho \\
\sigma \rho & \sigma^{2}+\sigma \frac{\omega_{1}}{\omega_{2}} \sqrt{1-\rho^{2}}
\end{array}\right) .
$$

The previous computations allow us to generalize to any return covariance matrix written $\Sigma=\left(\begin{array}{cc}\sigma_{1}^{2} & \rho \sigma_{1} \sigma_{2} \\ \rho \sigma_{1} \sigma_{2} & \sigma_{2}^{2}\end{array}\right)$ as then we have:

$$
\Lambda_{\mathrm{kyle}}=\frac{1}{\sqrt{\sigma_{1}^{2} \omega_{1}^{2}+2 \omega_{1} \omega_{2} \sigma_{1} \sigma_{2} \sqrt{1-\rho^{2}}+\sigma_{2}^{2} \omega_{2}^{2}}}\left(\begin{array}{cc}
\sigma_{1}^{2}+\frac{\omega_{2}}{\omega_{1}} \sigma_{1} \sigma_{2} \sqrt{1-\rho^{2}} & \sigma_{1} \sigma_{2} \rho \\
\sigma_{1} \sigma_{2} \rho & \sigma_{2}^{2}+\sigma_{1} \sigma_{2} \frac{\omega_{1}}{\omega_{2}} \sqrt{1-\rho^{2}}
\end{array}\right),
$$

which concludes the proof.

\section{B.2 Proof of Example 2}

Example 3 (Calendar Spread). Let $\Sigma:=\sigma^{2}\left(\begin{array}{ccc}1 & \rho & 1-\rho \\ \rho & 1 & -(1-\rho) \\ 1-\rho & -(1-\rho) & 2(1-\rho)\end{array}\right)$ and $\Omega:=\left(\begin{array}{ccc}\omega_{1}^{2} & 0 & 0 \\ 0 & \omega_{2}^{2} & 0 \\ 0 & 0 & \omega_{3}^{2}\end{array}\right)$. Then, one has:

$$
\Lambda_{\mathrm{ky} 1 \mathrm{e}}(\Sigma, \Omega)=\frac{\sigma \lambda_{1} \lambda_{2}}{6 \sqrt{\bar{\omega}_{1}^{2} \lambda_{1}^{2}+\bar{\omega}_{2}^{2} \lambda_{2}^{2}+2 \bar{\omega}_{1} \bar{\omega}_{2} \lambda_{1} \lambda_{2} \sqrt{1-\rho_{\omega}^{2}}}}\left(\begin{array}{ccc}
\frac{3 \lambda_{1}^{2}+\lambda_{2}^{2}}{\lambda_{1} \lambda_{2}}+\frac{3 \bar{\omega}_{2}^{2}+\bar{\omega}_{1}^{2}}{\bar{\omega}_{1} \bar{\omega}_{2} \sqrt{1-\rho_{\omega}^{2}}}+\frac{2 \sqrt{3} \rho_{\omega}}{\sqrt{1-\rho_{\omega}^{2}}} & \frac{3 \lambda_{1}^{2}-\lambda_{2}^{2}}{\lambda_{1} \lambda_{2}}+\frac{3 \bar{\omega}_{2}^{2}-\bar{\omega}_{1}^{2}}{\bar{\omega}_{1} \bar{\omega}_{2} \sqrt{1-\rho_{\omega}^{2}}} & 2 \frac{\lambda_{2}}{\lambda_{1}}+2 \frac{\bar{\omega}_{1}}{\bar{\omega}_{2} \sqrt{1-\rho_{\omega}^{2}}}+\frac{2 \sqrt{3} \rho_{\omega}}{\sqrt{1-\rho_{\omega}^{2}}} \\
\frac{3 \lambda_{1}^{2}-\lambda_{2}^{2}}{\lambda_{1} \lambda_{2}}+\frac{3 \bar{\omega}_{2}^{2}-\bar{\omega}_{1}^{2}}{\bar{\omega}_{1} \bar{\omega}_{\omega} \sqrt{1-\rho_{\omega}^{2}}} & \frac{3 \lambda_{1}^{2}+\lambda_{2}^{2}}{\lambda_{1} \lambda_{2}}+\frac{3 \bar{\omega}_{2}^{2}+\bar{\omega}_{1}^{2}}{\bar{\omega}_{1} \bar{\omega}_{2} \sqrt{1-\rho_{\omega}^{2}}}-\frac{2 \sqrt{3} \rho_{\omega}}{\sqrt{1-\rho_{\omega}^{2}}}-2 \frac{\lambda_{2}}{\lambda_{1}}-2 \frac{\bar{\omega}_{1}}{\bar{\omega}_{2} \sqrt{1-\rho_{\omega}^{2}}}+\frac{2 \sqrt{3} \rho_{\omega}}{\sqrt{1-\rho_{\omega}^{2}}} \\
2 \frac{\lambda_{2}}{\lambda_{1}}+2 \frac{\bar{\omega}_{1}}{\bar{\omega}_{2} \sqrt{1-\rho_{\omega}^{2}}}+\frac{2 \sqrt{3} \rho_{\omega}}{\sqrt{1-\rho_{\omega}^{2}}} & -2 \frac{\lambda_{2}}{\lambda_{1}}-2 \frac{\bar{\omega}_{1}}{\bar{\omega}_{2} \sqrt{1-\rho_{\omega}^{2}}}+\frac{2 \sqrt{3} \rho_{\omega}}{\sqrt{1-\rho_{\omega}^{2}}} & 4 \frac{\lambda_{2}}{\lambda_{1}}+4 \frac{\bar{\omega}_{1}}{\bar{\omega}_{2} \sqrt{1-\rho_{\omega}^{2}}}
\end{array}\right) .
$$

where $\rho_{\omega}:=\frac{\omega_{1}^{2}-\omega_{2}^{2}}{\sqrt{\left(\omega_{1}^{2}+\omega_{2}^{2}\right)\left(\omega_{1}^{2}+\omega_{2}^{2}+4 \omega_{3}^{2}\right)}}$ and $\bar{\omega}_{1}^{2}:=\frac{\omega_{1}^{2}+\omega_{2}^{2}}{2}, \bar{\omega}_{2}^{2}:=\frac{\omega_{1}^{2}+\omega_{2}^{2}+4 \omega_{3}^{2}}{6}, \lambda_{1}=\sqrt{1+\rho}, \lambda_{2}=\sqrt{1-\rho}$. Furthermore,

$$
\Lambda_{\mathrm{el}}(\Sigma, \Omega)=\sqrt{1+\rho} \frac{\sigma}{\bar{\omega}_{1}}\left(\begin{array}{lll}
1 & 1 & 0 \\
1 & 1 & 0 \\
0 & 0 & 0
\end{array}\right)+\sqrt{1-\rho} \frac{\sigma}{\bar{\omega}_{2}}\left(\begin{array}{ccc}
1 & -1 & 2 \\
-1 & 1 & -2 \\
2 & -2 & 4
\end{array}\right) .
$$


By construction, the two legs and the calendar spread are linearly related, therefore with a proper change of basis (thanks to the invariance of basis, see Axioms 4 and 5 one can reduce this three-dimensional case to Example 1. The return covariance matrix can be diagonalized as

$$
\begin{aligned}
\Sigma & =\sigma^{2}\left(\begin{array}{ccc}
1 / \sqrt{2} & \sqrt{1 / 6} & -\sqrt{1 / 3} \\
1 / \sqrt{2} & -\sqrt{1 / 6} & \sqrt{1 / 3} \\
0 & \sqrt{2 / 3} & \sqrt{1 / 3}
\end{array}\right)\left(\begin{array}{ccc}
1+\rho & 0 & 0 \\
0 & 1-\rho & 0 \\
0 & 0 & 0
\end{array}\right)\left(\begin{array}{ccc}
1 / \sqrt{2} & \sqrt{1 / 6} & -\sqrt{1 / 3} \\
1 / \sqrt{2} & -\sqrt{1 / 6} & \sqrt{1 / 3} \\
0 & \sqrt{2 / 3} & \sqrt{1 / 3}
\end{array}\right)^{-1} \\
& =: \sigma^{2} P \Sigma_{D} P^{-1} .
\end{aligned}
$$

By the invariance of basis, we have:

$$
\Lambda_{\mathrm{kyle}}(\Sigma, \Omega, R)=P \Lambda_{\mathrm{kyle}}\left(\Sigma_{D}, P^{-1} \Omega P, P^{-1} R P\right) P^{-1} .
$$

Furthermore, using fragmentation invariance (see Axioms 8 and 10, the impact matrix is left unchanged if the volume covariance's projection along zero-risk modes is set to zero. Thus, denoting $\Pi$ the projection orthogonal to zero risk modes, we obtain:

$$
\Lambda_{\mathrm{kyle}}(\Sigma, \Omega, R)=P \Lambda_{\mathrm{kyle}}\left(\Sigma_{D}, \Pi P^{-1} \Omega P \Pi, P^{-1} R P\right) P^{-1} .
$$

Thus, our computations reduce to computing the Kyle model on a 2-dimensional system of covariances with diagonal $\Sigma$ and non-diagonal $\Omega$. To do so, note first that $\Lambda_{\mathrm{kyle}}$ is symmetric and:

$$
\Lambda_{\mathrm{kyle}}(\Sigma, \Omega, R)=\mathscr{G} O \mathscr{L}^{-1}
$$

with $O=\mathscr{R} \mathscr{G} \sqrt{(\mathscr{R} \mathscr{G})(\mathscr{R} \mathscr{G})^{T}}$. Thus, we can use symmetry and:

$$
\Lambda_{\mathrm{kyle}}(\Sigma, \Omega, R)=\mathscr{G} O \mathscr{L}^{-1}=\mathscr{L}^{-T} O^{T} \mathscr{G}^{T}=\mathscr{L}^{-T} O^{T} \mathscr{G}=\Lambda_{\mathrm{kyle}}\left(\Omega^{-1}, \Sigma, R\right) .
$$

Here, as:

$$
\Pi P^{-1} \Omega P \Pi=\left(\begin{array}{ccc}
\frac{\omega_{1}^{2}+\omega_{2}^{2}}{2} & \frac{\omega_{1}^{2}-\omega_{2}^{2}}{2 \sqrt{3}} & 0 \\
\frac{\omega_{1}^{2}-\omega_{2}^{2}}{2 \sqrt{3}} & \frac{\omega_{1}^{2}+\omega_{2}^{2}+4 \omega_{3}^{2}}{6} & 0 \\
0 & 0 & 0
\end{array}\right)
$$

writing $\rho_{\omega}:=\frac{\omega_{1}^{2}-\omega_{2}^{2}}{\sqrt{\left(\omega_{1}^{2}+\omega_{2}^{2}\right)\left(\omega_{1}^{2}+\omega_{2}^{2}+4 \omega_{3}^{2}\right)}}$ and $\bar{\omega}_{2}^{2}:=\frac{\omega_{1}^{2}+\omega_{2}^{2}+4 \omega_{3}^{2}}{6}, \bar{\omega}_{1}^{2}:=\frac{\omega_{1}^{2}+\omega_{2}^{2}}{2}$ and using Example 1 , we have:

$$
\Lambda_{\mathrm{kyle}}=\frac{\sigma \lambda_{1} \lambda_{2}}{\sqrt{\bar{\omega}_{1}^{2} \lambda_{1}^{2}+\bar{\omega}_{2}^{2} \lambda_{2}^{2}+2 \bar{\omega}_{1} \bar{\omega}_{2} \lambda_{1} \lambda_{2} \sqrt{1-\rho_{\omega}^{2}}}}\left(\begin{array}{cc}
\frac{\lambda_{1}}{\lambda_{2}}+\frac{\bar{\omega}_{2}}{\bar{\omega}_{1} \sqrt{1-\rho_{\omega}^{2}}} & \begin{array}{c}
\frac{\rho_{\omega}}{\sqrt{1-\rho_{\omega}^{2}}} \\
\frac{\rho_{\omega}}{\sqrt{1-\rho_{\omega}^{2}}}
\end{array} \frac{\lambda_{2}}{\lambda_{1}}+\frac{\bar{\omega}_{1}}{\bar{\omega}_{2} \sqrt{1-\rho_{\omega}^{2}}}
\end{array} .\right.
$$

So that finally, moving back to the original basis:

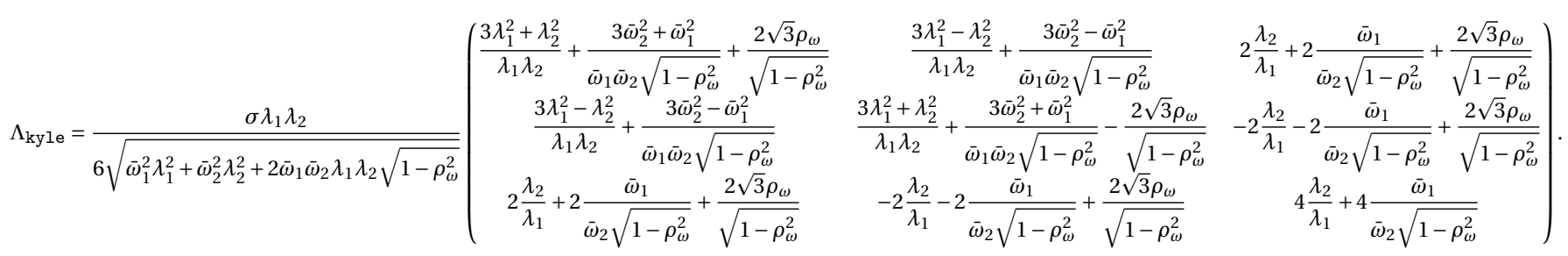

